\title{
TU/e EmonONEN

\section{A broadband method of quantifying phase synchronization for discriminating seizure EEG signals}

\section{Citation for published version (APA):}

Wang, L., Long, X., Aarts, R. M., van Dijk, J. P., \& Arends, J. B. A. M. (2019). A broadband method of quantifying phase synchronization for discriminating seizure EEG signals. Biomedical Signal Processing and Control, 52, 371-383. https://doi.org/10.1016/j.bspc.2018.10.019

\section{Document license:}

TAVERNE

DOI:

10.1016/j.bspc.2018.10.019

Document status and date:

Published: 20/06/2019

\section{Document Version:}

Publisher's PDF, also known as Version of Record (includes final page, issue and volume numbers)

\section{Please check the document version of this publication:}

- A submitted manuscript is the version of the article upon submission and before peer-review. There can be important differences between the submitted version and the official published version of record. People interested in the research are advised to contact the author for the final version of the publication, or visit the $\mathrm{DOI}$ to the publisher's website.

- The final author version and the galley proof are versions of the publication after peer review.

- The final published version features the final layout of the paper including the volume, issue and page numbers.

Link to publication

\section{General rights}

Copyright and moral rights for the publications made accessible in the public portal are retained by the authors and/or other copyright owners and it is a condition of accessing publications that users recognise and abide by the legal requirements associated with these rights.

- Users may download and print one copy of any publication from the public portal for the purpose of private study or research.

- You may not further distribute the material or use it for any profit-making activity or commercial gain

- You may freely distribute the URL identifying the publication in the public portal.

If the publication is distributed under the terms of Article 25fa of the Dutch Copyright Act, indicated by the "Taverne" license above, please follow below link for the End User Agreement:

www.tue.nl/taverne

Take down policy

If you believe that this document breaches copyright please contact us at:

openaccess@tue.nl

providing details and we will investigate your claim. 


\title{
A broadband method of quantifying phase synchronization for discriminating seizure EEG signals
}

\author{
Lei Wang ${ }^{\mathrm{a}, \mathrm{c}, *}$, Xi Long $^{\mathrm{a}, \mathrm{b}, *}$, Ronald M. Aarts ${ }^{\mathrm{a}, \mathrm{b}}$, Johannes P. van Dijk ${ }^{\mathrm{a}, \mathrm{c}}$, \\ Johan B.A.M. Arends ${ }^{\mathrm{a}, \mathrm{c}}$ \\ a Department of Electrical Engineering, Eindhoven University of Technology, Eindhoven, the Netherlands \\ b Philips Research, Eindhoven, the Netherlands \\ ${ }^{\mathrm{c}}$ Epilepsy Center Kempenhaeghe, Heeze, the Netherlands
}

\section{A R T I C L E I N F O}

\section{Article history:}

Received 18 March 2018

Received in revised form 1 October 2018

Accepted 22 October 2018

Available online 13 November 2018

\section{Keywords:}

Phase synchronization (PS)

Correlation between probabilities of

recurrence (CPR)

Phase lock index (PLI)

Epilepsy, intellectual disability

EEG discharge patterns

\begin{abstract}
A B S T R A C T
The nonlinear nature of phase coupling enables rich and context-sensitive interactions that characterize real brain dynamics, playing an important role in brain dysfunction such as epileptic disorders. Numerous phase synchronization (PS) measurements have been developed for seizure detection and prediction. However, the performance remains low for minor seizures in epileptic patients with an intellectual disability (ID), who are characterized by complex EEG signals associated with brain development disorders. The traditional PS measurements, e.g., phase locking index (PLI), are limited by the inability in detecting the nonlinear coupling of EEG signals and are sensitive to the background noise. This study focuses on developing a new EEG feature that can measure the nonlinear coupling, which thus would help improve seizure detection performance. We employ the correlation between probabilities of recurrence (CPR) to measure the PS on broadband EEG signals. CPR can capture the underlying nonlinear coupling of EEG signals and is robust to signal frequency and amplitude variance. The effectiveness of CPR-based features on identifying seizure EEG was evaluated on 26 epileptic patients with ID. Results show that the PS changes in seizures depend on the EEG discharge patterns including fast spike (SP), spike-wave (SPWA), wave (WA) and discharge with EMG activity (EMG). CPR-based PS decreased significantly in seizures with SP and EMG, (-0.1845 and -0.4278 , with 95\% CI [-0.1850, -0.1839] and [-0.4283, -0.4273], respectively), while it increases significantly in the SPWA seizures (+0.0746, with 95\% CI [0.0744, 0.0749]). In addition, CPR-based PS shows potential for predicting SPWA and EMG seizures in an early manner. We conclude that CPR measurement is promising to improve seizure detection in ID patients and provides a promising method for modeling epilepsy-related brain functional networks.
\end{abstract}

(C) 2018 Elsevier Ltd. All rights reserved.

\section{Introduction}

Phase synchronization (PS) is often used to measure the functional connectivity between different brain areas, based on electroencephalography (EEG), electrocorticography (ECoG), magnetoencephalography (MEG), and functional magnetic resonance imaging (fMRI) [1,2]. PS plays an important role in characterizing brain dysfunction such as epileptic disorders [3]. There is a variety of methods of quantifying PS between two time-series signals. Traditional PS measurements such as phase locking index [4], synchronization likelihood [2] and other linear coherence including

\footnotetext{
* Corresponding authors at: Eindhoven University of Technology, Eindhoven, the Netherlands.

E-mail addresses: Lei.Wang@tue.nl (L. Wang), xi.long@philips.com (X. Long).
}

cross-correlation [5] and (cross-)spectral densities [6] have been well studied. It has been found that these methods achieve similar efficacy in seizure detection and prediction [7-10], because they capture mainly the frequency-domain information and characterize the linear phase coupling of EEG signals. However, the nonlinear nature of phase coupling also plays an important role in functional integration and characterize real brain dynamics [11]. EEG signals originate from a spatial integration of post-synaptic potentials, which can be viewed as a nonlinear system consisting of numerous neuron activities. The chaotic behavior (i.e., a nonlinear property) of the brain has been observed in the EEG signals of healthy people $[12,13]$. The normal and epileptic EEG signals show different chaotic behaviors [14]. The evidence of chaotic behavior, the presence of an infinite number of unstable periodic fixed points has been found in epilepsy-related, spontaneously neuronal networks 

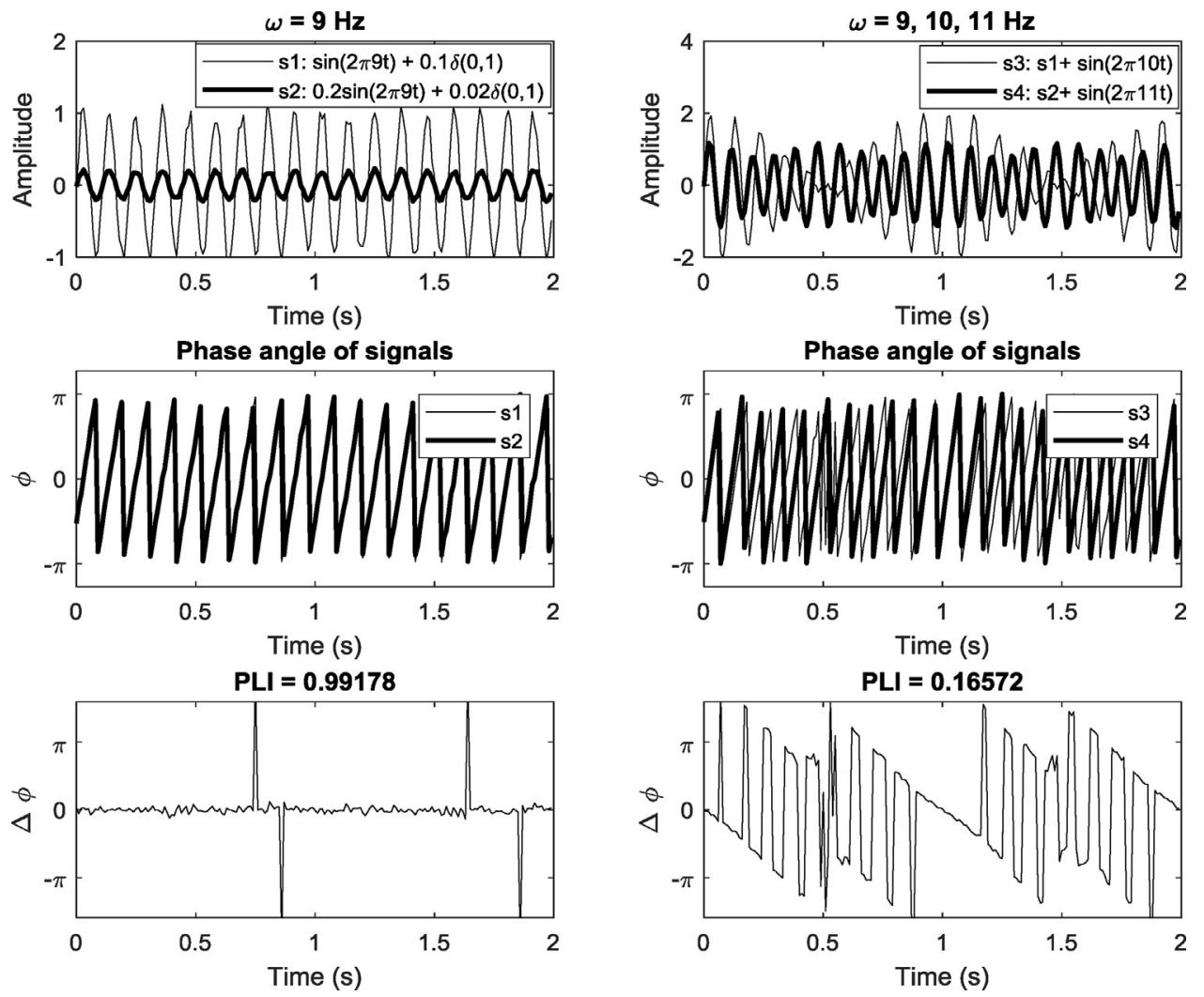

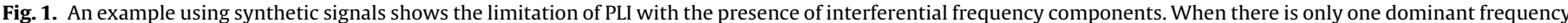

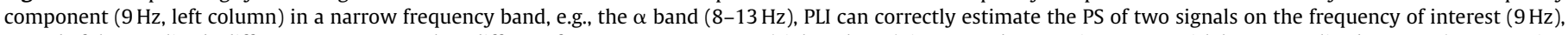

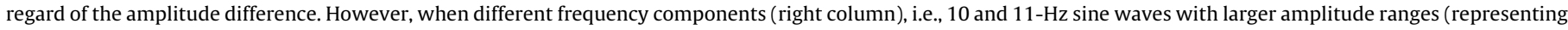
background EEG activities in the $\alpha$ band) were added, PLI fails to represent the PS on the frequency component of interest (9 Hz).

[15]. Therefore, the nonlinear properties of EEG are expected to be promising for seizure detection.

As a typical example of traditional linear methods, the phase locking index (PLI) and extended methods have been widely used for seizure detection $[16,17]$ and even prediction $[5,18,19]$. PLI analyzes EEG signals in the frequency domain and takes only the instantaneous phases of narrow-band signals into account, regardless of uncorrelated amplitudes [4]. Many methods such as PLI need to be performed in pre-defined, narrow-band signals [1]. As a result, PLI has a limitation that it may fail to represent the PS of frequency components of interest without a prior knowledge about the target frequency (band), which is demonstrated in Fig. 1. However, such a prior knowledge is often impossible because the frequency components related to seizures may vary across individuals and EEG recordings. Furthermore, PLI is limited by the inability to characterize the nonlinear properties of EEG signals.

Automated EEG-based seizure detection [20] has been well studied in populations such as patients with temporal lobe epilepsy $[21,7]$. However, it has not yet been sufficiently studied in the population with intellectual disability (ID) [22,23]. The reasons are twofold. Firstly, long-term EEG recordings are difficult to collect due to behavioral problems. Secondly, manual seizure annotation based on EEG recordings for this specific patient group is often difficult due to the complex EEG signal abnormalities caused by brain development disorders [24,25]. As a result, the performance of automated seizure detection for ID patients remains unclear. Our previous study [26] shows that the automated detection is difficult for minor seizures that show blurry boundaries associated with abnormal background EEG signals. The traditional EEG feature-based seizure detection methods $[20,22,27]$ may have limitations on detecting these minor seizures because they do not
Table 1

Patient demographics.

\begin{tabular}{lll}
\hline Parameters & Mean \pm Std & Range \\
\hline Gender & 14 males and 12 females \\
ID level $^{\mathrm{a}}$ & 3 light, 10 moderate and 13 severe \\
EEG discharge patterns $^{\mathrm{b}}$ & $7 \mathrm{SP}, 7 \mathrm{SPWA}, 8 \mathrm{~W}$ A and 8 EMG \\
Historical seizures $^{\mathrm{c}}$ & $21 \mathrm{GTC}, 9 \mathrm{MS}$, and 11 F & \\
Seizure number $_{\text {AT }^{\mathrm{d}} \text { of seizure [sec] }}$ & $4 \pm 2$ & $2-13$ \\
EEG recording length [hrs] $_{\text {Age [yrs] }}$ & $112 \pm 173$ & $7-709$ \\
& $22.3 \pm 1.8$ & $17.4-25.8$ \\
\hline
\end{tabular}

a Severe [0-30], moderate [30-50] and light [50-70].

b Four patients show more than one dominate discharge pattern.

c Historical seizures record the historical seizures types of patients. $\mathrm{GTC}=$ generalized tonic-clonic seizure, $\mathrm{MS}=$ myoclonic seizure, $\mathrm{F}=$ focal seizure . Note that one patient can have more than one seizure type.

d Accumulated time (AT).

consider the underlying nonlinear interaction amongst EEG signals and the complex brain networks based on the nonlinear neuronal interconnectivity [28]. Therefore, this study focuses on developing and analyzing new EEG features that can quantify the nonlinear coupling, which thus would help improve seizure detection performance. Note that, from detection perspectives, the classifier ensemble [29] and deep learning techniques [30] are promising in seizure detection, which is out of the scope of this work, however.

In this study, to detect the underlying nonlinear PS change related with seizures without using a prior knowledge of the frequency band information, we proposed to use the correlation between probabilities of recurrence (CPR) [31,32] (or synchronization index based on recurrence probabilities in [33]) to quantify PS. It has been shown that CPR is able to capture the underlying nonlinear coupling between two signals when strongly corrupted by noise 


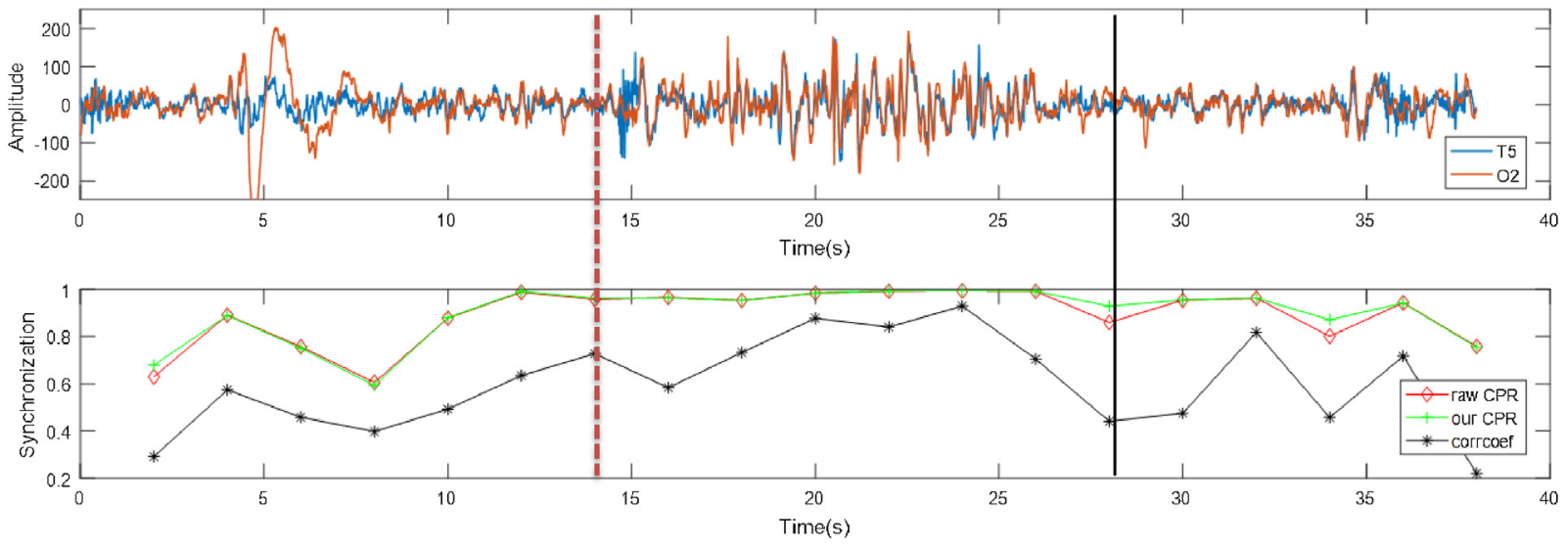

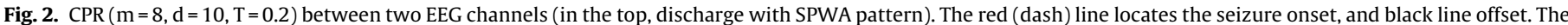

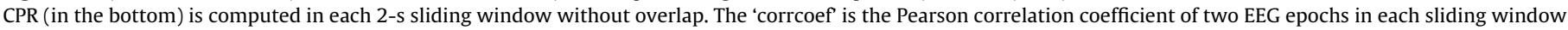
and is plotted here as a reference.

[34], [35], and CPR indicates clearly the onset of PS (or different synchronization status) [33]. The method to infer synchronized status with an ambiguous range of PS has been further developed [36]. CPR has been used on EEG [31], and fMRI data to recognize brain connectivity [37]. Compared with PLI, CPR does not need to filter the EEG to obtain narrow-band signals, which therefore can be considered as a broadband method [38]. Furthermore, it has been experimentally demonstrated that CPR outperforms the Hilbert transform in terms of robustness on non-stationary data [39]. This is because CPR is not a frequency-domain method and is less sensitive to spectrum change of signals caused by noise. These properties make CPR suitable to analyze EEG signals. We refer mathematical details and examples with regard to the comparison between CPR and Hilbert transform to [39].

To evaluate the effectiveness of CPR-based methods on detecting seizure EEG patterns for ID patients, we computed the CPR-based mean PS and compared it with PLI. We also investigated whether it is possible to predict seizures in an early manner (prior to the seizure onsets) by analyzing separability between background EEG and pre-seizure EEG.

- Instead of treating all seizure types as an entity (a common pitfall), we evaluated seizures according to EEG discharge patterns due to the heterogeneous EEG characteristics of the ID population [26].

- Instead of using manually-selected, artifact-free EEG sessions (often from long-term interictal recordings), we used randomlyselected, continuous EEG sessions to represent real-life interictal EEG signals.

- We compared CPR with a traditional method PLI on the same dataset.

- We optimized the computation of CPR to reduce the computation costs and variance across EEG epochs.

- We took into account the effect (i.e., different baselines of PS) of the asleep and awake status on the EEG characteristics.

This rest of the paper is organized as follows. Section 2 describes patient selection, EEG dataset, and preprocessing methods, then introduces our proposed method (CPR), reviews a traditional method (PLI) and proposes a PS measurement, and explains the statistical analysis methods to use. Section 3 presents sampled EEG data, CPR- and PLI-based PS results. Section 4 compares and interprets the results of the two methods and indicates clinical relevance. Finally, in Section 5, our main conclusions are drawn.

\section{Materials and methods}

\subsection{Patient selection}

As this study aims to evaluate a new method of quantifying PS for the application of seizure detection and prediction in the ID population, we need to employ all possible morphologies of seizure EEG signals (i.e., EEG discharge patterns). In our previous studies $[22,27]$, we analyzed four major EEG discharge patterns in the ID population: fast spike (SP), spike-wave (SPWA), wave (WA) and discharge with EMG activity (EMG). The seizure detection performance varies substantially with these EEG discharge patterns. In this study, we performed a retrospective data collection from data archive recorded from 2008 to 2017 in the Epilepsy Center Kempenhaeghe. The ID patients who showed at least one EEG seizure (i.e., showing the visual EEG change during seizure events checked by a neurologist) in continuous 24-hour ambulatory EEG recordings were included. Seizures without EEG change were excluded due to the lack of timing information for annotation. Since it is often not allowed to record video at the patients' home. No synchronous video could be used for annotation. The EEG experts excluded uncertain seizures or non-seizure EEG, which may undermine the statistical analysis results. In the future work, the performance evaluation of seizure detection on a larger dataset, we will use all the seizure events (certain or uncertain) to avoid a source bias. In this work, the total excluded uncertain seizure EEG epochs were less than $5 \%$ of all seizure EEG epochs. Therefore, it has little effect on the reported results.

We finally selected 26 patients with approximate numbers of patients for each EEG discharge pattern. The patient demographics are shown in Table 1. The study was approved by Kempenhaeghe's ethical review board.

\subsection{EEG data E’ annotations}

Continuous scalp EEG signals (sampling rate of $100 \mathrm{~Hz}$ ) were acquired using $24 \mathrm{~A} \mathrm{G} /$ AGCL electrodes according to the 10-20 positioning system. Recordings were performed at home without video with an ambulant EEG system (Porti 5, Twente Medical Systems International) and reviewed using the BrainRT ${ }^{\mathrm{TM}}$ software.

Clinical information about the seizures was retrieved from the diaries provided by caregivers, medical history, and the final EEG reports. A stepwise EEG annotation procedure was preferred above a simple one-step approach with an inter-observer agreement test. First, the EEG seizures were annotated by EEG technicians when preparing the EEG report. In a second step, all EEGs were re- 


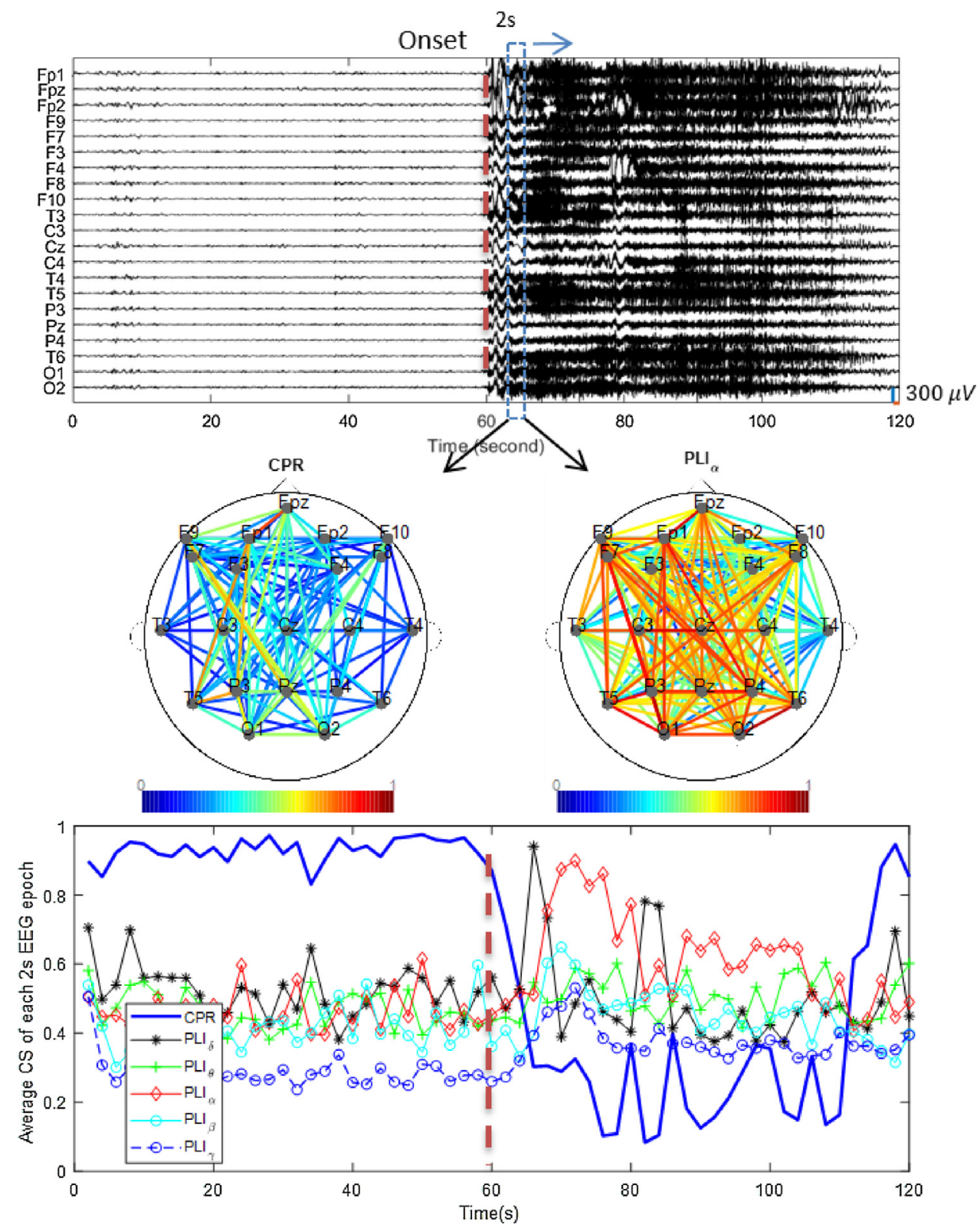

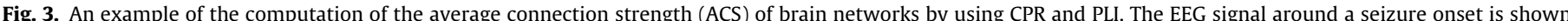

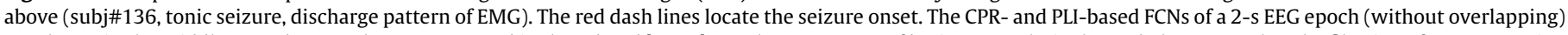

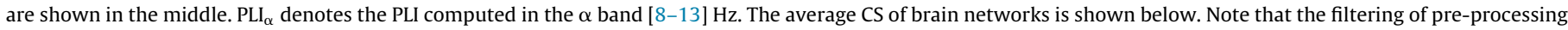
caused the distortion at the beginning of the EEG segment, which results in the high values of PLI at the first point.

annotated by a clinical neurophysiologist specialized in epilepsy. These re-annotations formed the basis of the final selection of EEG data and included the onset and offset of EEG seizures and the EEG discharge patterns. During an EEG seizure, the mixed EEG discharge patterns (i.e., polyspike complexes) were annotated by the dominant pattern in time. We also annotated patients' awake and sleep status that would potentially cause differences in EEG signals [40]. Due to the lack of automated classification methods of sleep/awake status in the ID population, the awake and sleep status was estimated from the diaries and the classification of the sleep differentiation by the EEG technicians.

\subsection{EEG preprocessing}

Unipolar montage is used for the EEG measurement to avoid changing the synchrony among EEG channels [41]. Firstly, on each EEG channel, the signals are filtered by using a 10th-order But- terworth bandpass filter with the lower and the higher cutoff frequency of $0.5 \mathrm{~Hz}$ and $45 \mathrm{~Hz}$, respectively. Secondly, EEG channel selection has been performed to choose the channels that contain EEG with good signal quality. In each non-overlapping sliding window of two seconds, we keep only the channels in which the EEG epoch is within amplitude range (i.e., half of peak-to-peak amplitude) $[10-200] \mu \mathrm{V}$ for further analysis. The lower boundary $(10 \mu \mathrm{V})$ was to reject artifacts caused by loose electrode-skin collection or sweating. The higher boundary $(200 \mu \mathrm{V})$ was to reject excessive artifacts caused by movements, electrocardiogram (ECG), and excessive EMG activities.

\subsection{Correlation between probabilities of recurrence (CPR)}

Synchronization of dynamical systems is a key nonlinear phenomenon, it has become increasingly important in recent nonlinear EEG analysis [34]. CPR measures the probabilities of phase recur- 
Table 2

Patient groups and number of sampled 2-s EEG epochs.

\begin{tabular}{|c|c|c|c|c|c|c|}
\hline $\begin{array}{l}\text { Seizure discharge } \\
\text { patterns }\end{array}$ & No. of Patients & No. of seizures & $\begin{array}{l}\text { No. of seizure } \\
\text { epochs }\end{array}$ & $\begin{array}{l}\text { No. of awake EEG } \\
\text { epochs }{ }^{a}\end{array}$ & $\begin{array}{l}\text { No. of sleep EEG } \\
\text { epochs }^{\text {a }}\end{array}$ & $\begin{array}{l}\text { No. of pre-seizure EEG } \\
\text { epochs }^{\text {b }}\end{array}$ \\
\hline SP & 7 & 9 & 85 & 12,600 & 12,600 & 270 \\
\hline SPWA & 7 & 43 & 250 & 12,600 & 12,600 & 1290 \\
\hline WA & 8 & 17 & 525 & 14,400 & 14,400 & 510 \\
\hline EMG & 8 & 21 & 568 & 14,400 & 14,400 & 630 \\
\hline
\end{tabular}

a Six continuous 10-min EEG segments are randomly sampled from each patient's awake or sleep background (non-seizure) EEG recordings.

b A continuous 1-min EEG segment is selected before each seizure onset.

Table 3

p-values, median difference and 95\% CI of CPR-based ACS.

\begin{tabular}{|c|c|c|c|c|}
\hline \multirow{2}{*}{$\begin{array}{l}\text { EEG epochs to } \\
\text { compare }^{*}\end{array}$} & \multicolumn{4}{|c|}{ Patient groups with EEG discharge patterns } \\
\hline & SP & SPWA & WA & EMG \\
\hline \multirow[t]{2}{*}{ Sleep vs. Awake } & $0^{* *}$ & 0 & 0 & 0 \\
\hline & $0.0540,[0.0540,0.0540]$ & $0.0871,[0.0870,0.0871]$ & $0.0467,[0.0467,0.0467]$ & $0.0439,[0.0438,0.0439]$ \\
\hline \multirow[t]{2}{*}{ SEZ vs. Non-SEZ } & $2.176 \mathrm{e}-28$ & $3.170 \mathrm{e}-37$ & $4.182 \mathrm{e}-07$ & $6.020 \mathrm{e}-212$ \\
\hline & $-0.1845,[-0.1850,-0.1839]$ & $0.0746,[0.0744,0.0749]$ & $-0.0207,[-0.0208,-0.0206]$ & $-0.4278,[-0.4283,-0.4273]$ \\
\hline \multirow[t]{2}{*}{ Pre-SEZ vs. Non-SEZ } & 0.179 & $3.243 e-39$ & 0.827 & $4.910 \mathrm{e}-06$ \\
\hline & $-0.0051,[-0.0051,-0.0050]$ & $0.0403,[0.0402,0.0404]$ & $0.0000,[0.0000,0.0000]$ & $0.0088,[0.0087,0.0088]$ \\
\hline
\end{tabular}

* The estimated median difference of two groups is computed by the former subtracting the latter (e.g., Sleep - Awake).

** 0 denotes $\mathrm{p}<1.000 \mathrm{e}-255$.

rence in two dynamic systems and then computes the correlation between two probability series. Its computation is not sensitive to neither signal frequency nor relative amplitudes of two signals. The time epoch showing a high value of CPR corresponds to the place where two dynamic trajectories approach a chaotic attractor [42], which may suggest a changed status associated with epileptic seizures.

Based on the chaos theory, a single time series $u(t)$ can be reconstructed into a series of vectors in a phase space by 'time-delay embedding' [43]. A vector represents a state of the system, such as

$\vec{x}_{i}=[u(i), u(i+d), \ldots, u(i+(m-1) d)]$

where $m$ denotes the embedding dimension and $d$ the time delay. If the embedding dimension $m$ is sufficiently high (more than twice the dimension of the system's attractor), the series of reconstructed vectors constitute an 'equivalent attractor', which has the same dynamical properties as the original attractor, [42]. However, a larger $m$ increases the computation costs, thus in practice, $m$ should be larger than a minimum embedding dimension and the optimal value depends on specific human brain signals [14,34]. In this work, based on Cao's method [14], we set $m=8$ (larger than the minimum embedding dimension of both seizure and non-seizure EEG segments), so that epileptic EEG signals can show chaos determinism. We then empirically set $d=10$, which is because that what really matters is the $m * d$ product, i.e., how much of the data are spanned by an embedding vector, and thus that estimating the two parameters in combination may be more effective [44]. In our case, the span of a vector is $800 \mathrm{~ms}$ for EEG signals with a sample rate of $100 \mathrm{~Hz}$. it means that a vector can keep whole morphological information of a seizure spike. More discussion about the choice of parameters can be found in [31].

The connection between successive vectors in a phase space defines the trajectory of a dynamical system. We define a recurrence of the trajectory as: the trajectory has returned at $i$ to the former state at $j$ if

$R_{i, j}^{m, \varepsilon}=\Theta\left(\varepsilon-|| \vec{x}_{i}-\vec{x}_{j} \mid\right)=1$

where $\vec{x}_{i}$ and $\vec{x}_{j}$ are trajectory vectors in an m-dimension phase space. $\Theta()$ is the Heaviside function, and \||| denotes Euclidean norm. $\varepsilon$ is a pre-defined threshold, $\varepsilon=T \max _{i j}\left(\left|\vec{x}_{i}-\vec{x}_{j}\right|\right), T \in$ $(0,1)$ where the coefficient threshold $T \in(0,1)$. By varying this threshold $T$, we can trade between resolution and stationarity requirements [32]. We empirically choose $T=0.2$ to ensure a good resolution, i.e., high discriminative power between seizure and non-seizure EEG epochs. The value of $\varepsilon$ can change with an amplitude range of different signal sessions so that the recurrence $R_{i, j}^{m, \varepsilon}$ is independent of the amplitude scales of two signals. Based on this definition of recurrence, we estimate the probability of recurrence $P(\tau)$, i.e., the system returns to the neighborhood of a former state of the trajectory after $\tau$ time steps:

$P(\tau)=\frac{1}{N-\tau} \sum_{i=1}^{N-\tau} R_{i, i+\tau}^{m, \varepsilon}, \quad \tau \in\left[1, \tau_{m}\right]$

where $P(\tau)$ can be viewed as a statistical measure on how often original phase space has increased by (multiples of) $2 \pi$ within the time interval $\tau$ [45]. $\tau_{m}$ is the maximum range of $\tau, \tau_{m}=N-m+1$ [31]. If both systems are in PS, the probability of recurrence will be maximal at the same time. We can quantify the PS of two systems by measuring the similarity (or likelihood) of maxima of $P(\tau)$ for two systems. Firstly we compute the probabilities of recurrence $P_{1}(\tau)$ and $P_{2}(\tau)$ for the two EEG signals respectively. Secondly, we compute the correlation coefficient between probabilities of recurrences $(\mathrm{CPR})$ :

$C P R=\sum_{\tau=1}^{\tau_{m}} \frac{\left(P_{1}(\tau)-\overline{P_{1}}\right)\left(P_{2}(\tau)-\overline{P_{2}}\right)}{\sigma_{1} \sigma_{2}}$

where $\overline{P_{1}}, \overline{P_{2}}$ and $\sigma_{1}, \sigma_{2}$ are the mean value and standard deviation of $P_{1}(\tau)$ and $P_{2}(\tau)$, respectively. We found that in real EEG signals, the chance of recurrence within a shorter time interval is much larger than within a larger interval. The recurrence within a shorter time interval may be associated with a brain dynamic system, while the recurrence spanning a longer time interval may be simply caused by the periodicity of EEG signals, thereby causing noisy PS. Without omitting useful information, we thus choose the value of $\tau_{m}$ to be half of the length of a vector $\left(\tau_{m}=\frac{1}{2} N\right)$ to reduce the computation cost of CPR. In addition, our $\tau_{m}$ can reduce the variance of 


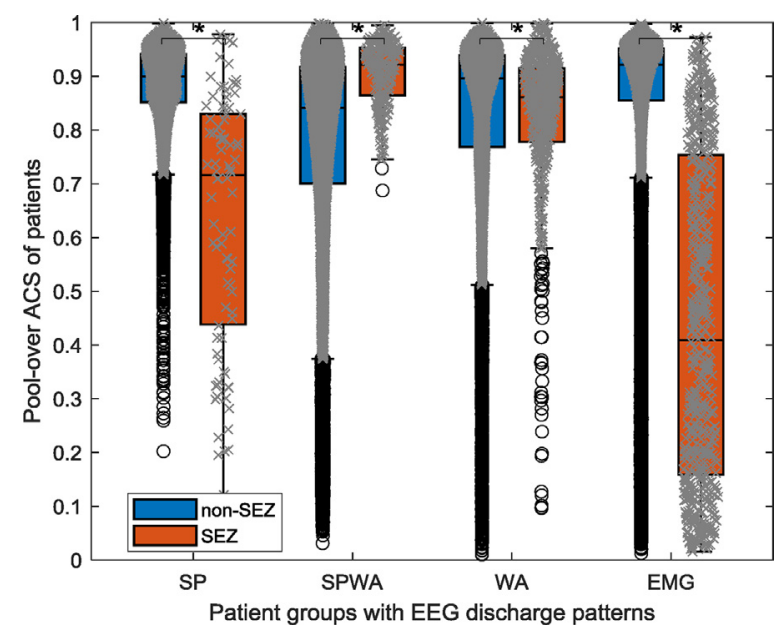

Fig. 4. Boxplot of pool-over ACS (CPR-based) of patients. Patients are divided into four groups according to the EEG discharge patterns: $S P\left(n_{0}=12600, n_{1}=85\right)$, SPWA $\left(\mathrm{n}_{0}=12600, \mathrm{n}_{1}=250\right)$, WA $\left(\mathrm{n}_{0}=14400, \mathrm{n}_{1}=525\right)$, and EMG $\left(\mathrm{n}_{0}=14400, \mathrm{n}_{1}=568\right)$, where $n_{0}$ is the size of non-seizure class (non-SEZ), $n_{1}$ is the size of seizure class (SEZ). The * denotes significant difference ( $p$ values refer to Table 2 ). The seizure EEG of the four patient groups are different (all $\mathrm{p}<1.0 \mathrm{e}-08$ ).

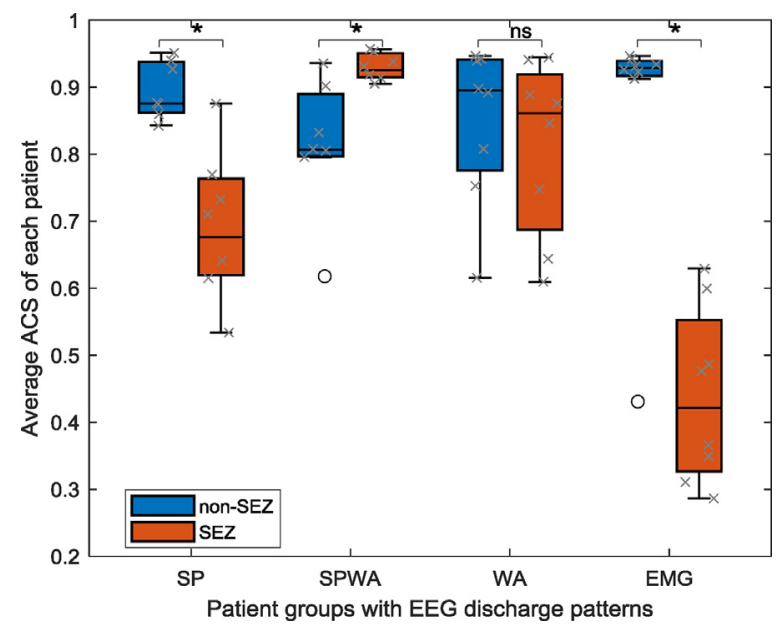

Fig. 5. Boxplot of average ACS (CPR-based) of each patient. Patients are divided into four groups according to the EEG discharge patterns: SP $(n=7)$, SPWA $(n=7)$, WA $(n=8)$ and EMG $(n=8)$. The * denotes significant difference; ns denotes not significant $(\mathrm{p}=0.0041,0.0070,0.4418$ and 0.0019 on groups SP, SPWA, WA and EMG respectively). The seizure EEG (SEZ) of the four patient groups are different (all $\mathrm{p}<0.05$ ) in arbitrary pairs except the pair between SP and WA.

CPR because noisy PS caused by periodicity is omitted (which can be characterized by FFT). An example of computing CPR between two EEG channels is shown in Fig. 2. Our CPR $\left(\tau_{m}=\frac{1}{2} N\right)$ achieves similar values with the raw CPR (using $\tau_{m}=N-m+1$ ), but with a smaller variance. Compared with Pearson's correlation coefficient (measuring the linear dependence two time series in a sliding window), CPR shows an increase of PS early before the seizure onset. EEG signals may show chaotic PS early before seizure onsets. Such chaotic PS shows nonlinear coherence between two EEG channels which is difficult for a linear method to detect. The nonlinear coherence finally evolves into a linear coherence during seizures. That is, CPR may be more sensitive to pre-seizure and post-seizure EEGs than the Pearson correlation coefficient because of the presence of the nonlinear interaction. See [33] for more details to exemplify this method.

\subsection{Phase lock index (PLI)}

PLI is also termed as phase synchronization index or phase locking value [4]. The computation of PLI is briefly described as follows. The instantaneous phase of a time series $x(t)$ can be calculated as

$\phi(t)=\arg (x(t)+i \hat{x}(t))$

where $\hat{x}(t)$ is the Hilbert transform of $x(t)$ [46]. The instantaneous phase can be estimated by using band-pass filters and then Hilbert's transform [47] or the complex wavelet method around a central frequency [19]. Hilbert's transform and the complex wavelet method were concluded to be fundamentally equivalent to the neuro-electrical signals analysis [19]. The difference of instantaneous phases between two signals $x_{a}(t)$ and $x_{b}(t)$ is defined as

$\Delta \phi_{a b}(t)=\phi_{a}(t)-\phi_{b}(t)$

which is a random variable characterized by a probability distribution. Hence, the strength of synchronization between two time series is quantified and termed as the phase lock index (PLI):

$P L I_{a b}=\left|\frac{1}{n} \sum_{t=1}^{n} \exp \left(i \Delta \phi_{a b}(t)\right)\right|$

In a duration of synchronized time series, $\Delta \phi_{a b}(t)$ is a constant, then $P L I_{a b}=1$. If the time series is unsynchronized, then $\Delta \phi_{a b}(t)$ follows a uniform distribution and $P L I_{a b}$ tend to be 0 . PLI can vary between 0 and 1 in real EEG signals.

Note that the instantaneous amplitude and phase of EEG signals have a clear physical meaning only in a narrow-band signal [45]. Therefore, in this study, we computed the PLI in the following five frequency bands: $\delta(0.5-4 \mathrm{~Hz}), \theta(4-8 \mathrm{~Hz}), \alpha(8-13 \mathrm{~Hz}), \beta$ $(13-30 \mathrm{~Hz})$, lower $\gamma(30-45 \mathrm{~Hz})[2]$.

\subsection{Average connecting strength (ACS)}

The result of computing the PS (by using PLI or CPR) for all pairwise EEG channels is a PS square matrix. We can directly map the PS matrix into a brain network, in which two EEG electrodes (nodes) are connected with an undirected edge with a connection strength between 0 and 1 . The mean value of connection strengths shows an overall intensity of the functional connection of brains. It is termed as the average connection strength (ACS). Since generalized seizures account for more than $97 \%$ of seizures while focal seizures less than $3 \%$ in the ID population [48], seizure EEG signals occur in all scalp EEG channels. Therefore, we evaluate the ACS during seizures.

Fig. 3 shows an example of the ACS during an EMG seizure computed by using CPR or PLI methods, separately. The CPR-based ACS decreases significantly during the seizure. In the EMG seizure, the real seizure EEG signals are overwhelmed by EMG artifacts, while CPR's parameters were chosen for tracking the chaotic attractors on EEG signals, CPR thus tends to show low values on the EMGdominant signals. However, PLI-based ACS increases on the $\alpha$ band and lower $\gamma$ band. It suggests that EMG seizures may still have frequency components that enter synchrony during seizures. Note that the increased value of ACS on the $\delta$ band may be caused by movement artifacts in a motor seizure, which should be ruled out as an evidence of seizures.

\subsection{Statistical analysis}

To represent the background EEG activities of patients within around $24 \mathrm{~h}$ EEG recordings, we randomly sampled six 10-min EEG segments from non-seizure EEG recordings during awake and sleep 

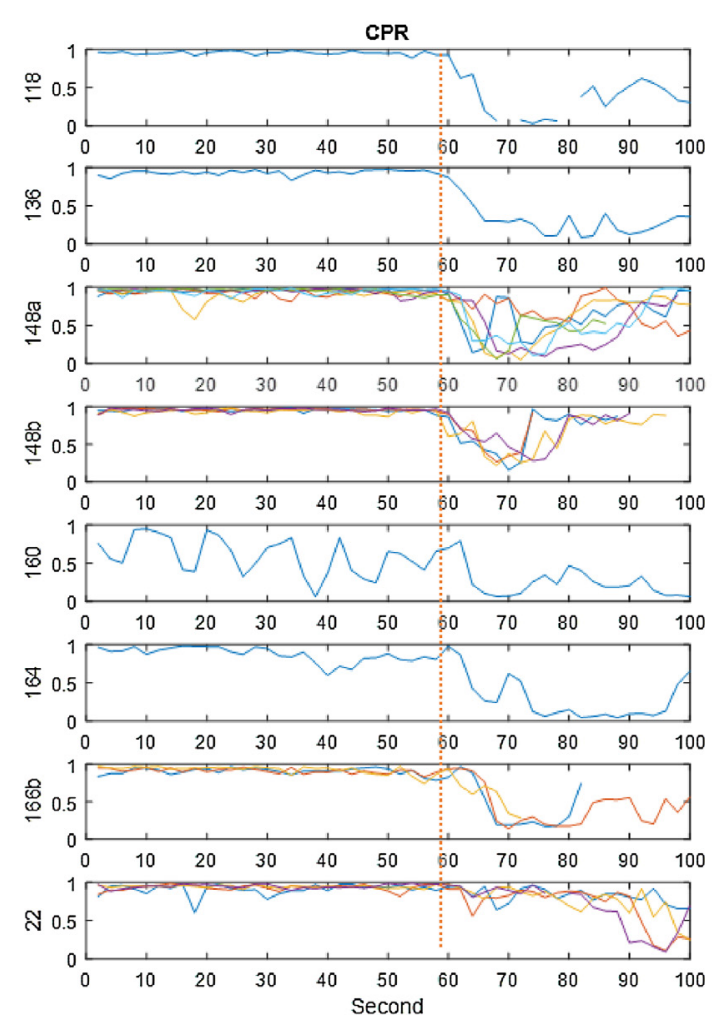
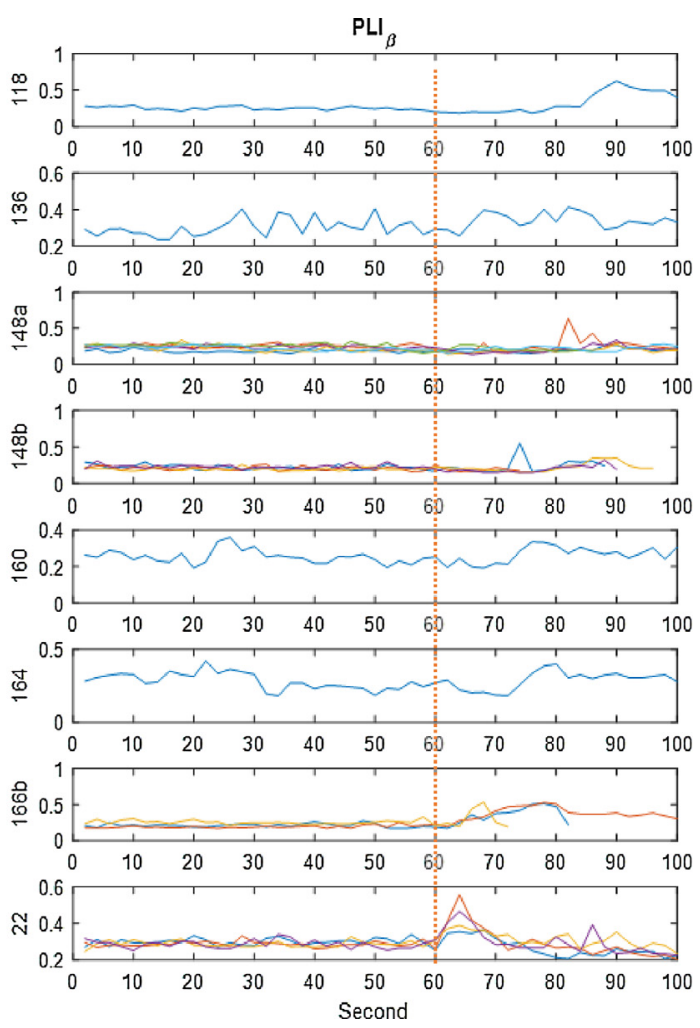

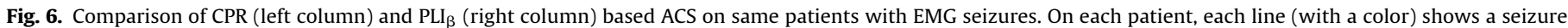
preceded by $60 \mathrm{~s}$ non-seizure EEG till seizure offsets. The dash lines show the aligned seizures onsets.

status, respectively. Specifically, we evenly divided non-seizure EEG recordings (excluding $30 \mathrm{~min}$. before seizure onset and $30 \mathrm{~min}$. after seizure offset) in an awake or asleep status into six parts, and randomly select a continuous 10 -min EEG segment with good signal quality from each part. The six 10-min EEG segments selected from an awake or sleep status were denoted as 'awake' or 'sleep' background. To avoid the influence of different baselines of EEG signals between an awake and a sleep status, we compared seizure and non-seizure EEG within either an awake or sleep status. That is, the non-seizure EEG segments were sampled from the same awake or sleep status where seizures occur. For each patient group (with a specific EEG discharge pattern), the background EEG (i.e., nonseizure EEG) during awake and sleep status were compared, and the accumulated seizure EEG and non-seizure EEG were compared.

Statistical tests were performed with the Mann-Whitney U test for unpaired data or a two-sided Wilcoxon signed-rank test for paired data $(\alpha<0.05)$. Note that the Mann-Whitney $U$ test and Wilcoxon rank sum test are mathematically equivalent tests (i.e., reporting same $\mathrm{p}$ values). Wilcoxon rank sum test statistic is equivalent to the area under the receiver operating characteristic (ROC) curve [49], which is often used to evaluate a binary classification performance between non-seizure and seizure EEG segments [5]. We can thus directly compare the p values (on the same dataset) to show the discriminative power between two EEG classes [50]. In addition to $\mathrm{p}$ values, we also reported the estimated median difference and its $95 \%$ confidence interval (CI). While it is often common to use a normal distribution approximation mean for a larger sample size $(N>20,000)$, however, to remain consistent across the presented results, we did not use such an approximation. Besides, our data does not follow a Gaussian distribution. Instead, we used the exact rank order to compute the median difference and 95\% CI. First, we computed all the possible differences between samples from the two groups. Second, we estimated the median difference as the value in the order of $0.5^{*} \mathrm{~N}\left(\mathrm{~N}=\mathrm{n} 1^{*} \mathrm{n} 2, \mathrm{n} 1\right.$ and $\mathrm{n} 2$ are sample sizes of two groups), and the $95 \% \mathrm{CI}$ is $\mathrm{N}^{*} 0.5 \pm 0.98^{*} \operatorname{sqrt}(\mathrm{N})$ rank value. This formula is based upon the assumption that the ranks of the patient samples are normally distributed.

\section{Results}

\subsection{Sampled data}

The data of the patients and EEG segments are shown in Table 2. EEG seizures shorter than $4 \mathrm{~s}$ are excluded. Four patients show two major EEG discharge patterns.

\subsection{CPR-based ACS}

We compared non-seizure EEG segments from the awake and sleep EEG recordings. The comparison was performed in patient groups with the same EEG discharge patterns. The CPR-based ACS shows significant differences between awake and sleep EEG backgrounds across all patient groups (see Table 3). The CPR-based PS in sleep is generally higher than that in awake status. The variance of ACS during awake status is larger than during sleep. This may be the result of more complicated brain functioning and more artifacts in awake status. The distributions of ACS in awake and sleep EEG are shown in Fig. B1 in Appendix B.

To evaluate the discriminative power of CPR-based ACS between seizure and non-seizure EEG, we performed statistical analysis on each patient group. For each patient group (i.e., seizure discharge pattern), we pool over the sampled seizure EEG and non-seizure EEG, separately. See the number of pool-over EEG epochs of each group in Table 2. We then compared the ACS between seizure and non-seizure EEG classes (see Fig. 4). The p values, medians, and 95\% $\mathrm{CI}$ are shown in Table 3. In addition, to show the variance across patients, we also averaged the ACS values of EEG epochs (seizure or 


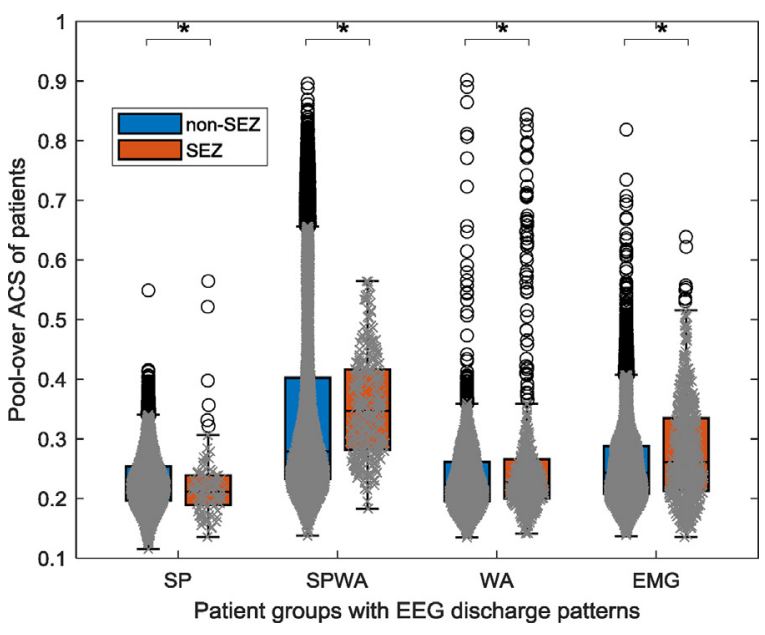

Fig. 7. Boxplot of PLI-based ACS ( $\beta$ band) on each patient group. Patients are divided into four groups according to the EEG discharge patterns: SP $\left(\mathrm{n}_{0}=12600, \mathrm{n}_{1}=85\right)$, SPWA $\left(\mathrm{n}_{0}=12600, \mathrm{n}_{1}=250\right)$, WA $\left(\mathrm{n}_{0}=14400, \mathrm{n}_{1}=525\right)$, and EMG $\left(\mathrm{n}_{0}=14400\right.$, $n_{1}=568$ ), where $n_{0}$ is the size of non-seizure class (non-SEZ), $n_{1}$ is the size of seizure class (SEZ). The * denotes significant difference between the seizure (SEZ) and non-seizure (non-SEZ) EEG ( $p$ values refer to Table 4).

non-seizure) on the same patients. The average ACS on each patient is shown in Fig. 5.

The pool-over ACS (Fig. 4) and average ACS (Fig. 5) show similar distributions, which suggests that the variance is largely determined by the across-patient difference. That is, the variance across patients is generally larger than within patients. Specifically, the pool-over ACS shows a significant difference between a seizure and non-seizure EEG across all patient groups, and the seizure EEG of the four patient groups are different (Mann-Whitney U test, all $\mathrm{p}<1.0 \mathrm{e}-08)$. ACS decrease in seizures with patterns of SP and EMG $(-0.18,-0.43$, respectively), while increases significantly $(+0.07)$ in seizures with a pattern of SPWA (see Table 3). The average ACS shows similar trends. However, the average ACS showed no significant difference between a seizure and non-seizure EEG in the group of WA. This may be due to a limited sample size of patients $(n=8)$. We show an example of dynamic ACS change during seizures in Fig. 6. More detailed ACS during seizures are shown in Appendix A.

The durations of pre-seizure EEG change may vary across patients from minutes to hours. In this study, we empirically evaluated only the pre-seizure EEG segments with $60 \mathrm{~s}$ before seizure onsets. We then compared ACS between the non-seizure and preseizure EEG segments to show the possibility of seizure prediction (longer than $60 \mathrm{~s}$ ). Table 3 shows that for the seizures with patterns of SPWA and EMG, non-seizure and pre-seizure EEGs are significantly different $(\mathrm{p}=3.243 \mathrm{e}-39$ and $4.910 \mathrm{e}-06$, respectively). The distributions of ACS in pre-seizure and seizure EEG on each patient group are shown in Fig. B2 of Appendix B.

\subsection{PLI-based ACS}

To evaluate the discriminative power of PLI-based ACS between non-seizure and seizure EEG, and between non-seizure and preseizure EEG, we compared the pool-over values of ACS in each patient group. Table 4 shows that both seizure detection and prediction are possible for the SPWA seizures on all frequency bands. PLI-based ACS in the $\beta$ band shows a significant difference (Non-SEZ vs. SEZ) in all patient groups (see Fig. 7). PLI on the $\beta$ band thus can be a good EEG feature for seizure detection. It is interesting to note that PLI may outperform CPR for seizure prediction (Non-SEZ vs. Pre-SEZ) on specific frequency bands (e.g., Lower $\gamma$ for SP and WA). It suggests that for PLI: (1) indicators of seizures could exist prior to the seizure EEG, and (2) the frequency bands need to be carefully chosen, which is often a posteriori procedure in a seizure detection task. In addition, similar to CPR, PLI-based ACS also shows significant differences between awake and sleep EEG backgrounds on all patient groups (all $\mathrm{p}<0.0001$ ). The estimated median difference and 95\% CI on PLI-based ACS can be found in Tables 5 and 6.

However, the average PLI-based ACS on each patient shows no significant difference between a seizure and non-seizure EEG in all groups except only one frequency band one EMG group (see Fig. 8). This is caused by a limited sample size of patients ( $n=7$ or 8) and a relatively large variance of PLI among each patient. In contrast, the average value of CPR can show a significant difference between a seizure and non-seizure EEG on three groups. Note that the value of PLI-based ACS is lower in high-frequency bands

Table 4

p values of Mann-Whitney $U$ test on PLI-based ACS.

\begin{tabular}{|c|c|c|c|c|c|c|c|c|c|}
\hline \multirow{2}{*}{$\begin{array}{l}\text { Frequency } \\
\text { bands }(\mathrm{Hz})\end{array}$} & & \multicolumn{4}{|c|}{ SEZ vs. Non-SEZ } & \multicolumn{4}{|c|}{ Pre-SEZ vs. Non-SEZ } \\
\hline & & SP & SPWA & WA & EMG & SP & SPWA & WA & EMG \\
\hline$\delta$ & {$[0.5,4]$} & 0.0010 & $9.65 \mathrm{e}-22$ & 0.3695 & $4.68 \mathrm{e}-35$ & 0.0016 & $2.50 \mathrm{e}-24$ & 0.0712 & $2.53 e-07$ \\
\hline$\theta$ & {$[4,8]$} & 0.0524 & $2.16 e-06$ & $2.35 \mathrm{e}-07$ & $1.00 \mathrm{e}-35$ & 0.0036 & $1.64 \mathrm{e}-36$ & 0.0625 & 0.1436 \\
\hline$\alpha$ & {$[8,13]$} & 0.2022 & $1.16 \mathrm{e}-09$ & 0.0687 & 0.2048 & $5.86 e-05$ & $4.91 \mathrm{e}-58$ & 0.0279 & 0.3666 \\
\hline$\beta$ & {$[13,30]$} & 0.0172 & $3.95 e-12$ & 0.0105 & $3.77 e-08$ & $1.56 \mathrm{e}-10$ & $4.62 e-33$ & 0.0877 & 0.0700 \\
\hline Lower $\gamma$ & {$[30,45]$} & 0.4660 & $2.50 \mathrm{e}-05$ & $5.23 e-45$ & $1.21 \mathrm{e}-09$ & $1.80 \mathrm{e}-09$ & $8.74 \mathrm{e}-05$ & 0.0001 & 0.0595 \\
\hline
\end{tabular}

Table 5

Median difference and 95\% CI between seizure and non-seizure EEG.

\begin{tabular}{|c|c|c|c|c|c|}
\hline \multirow{2}{*}{$\begin{array}{l}\text { Frequency } \\
\text { bands }(\mathrm{Hz})\end{array}$} & & \multicolumn{4}{|l|}{ SEZ vs. Non-SEZ } \\
\hline & & SP & SPWA & WA & EMG \\
\hline$\delta$ & {$[0.5,4]$} & $\begin{array}{l}0.0304, \\
{[0.0301,0.0304]}\end{array}$ & $\begin{array}{l}0.0519, \\
{[0.0517,0.0521]}\end{array}$ & $\begin{array}{l}-0.0028, \\
{[-0.0029,-0.0027]}\end{array}$ & $\begin{array}{l}-0.0399, \\
{[-0.0400,-0.0398]}\end{array}$ \\
\hline$\theta$ & {$[4,8]$} & $\begin{array}{l}-0.0131, \\
{[-0.0133,-0.0130]}\end{array}$ & $\begin{array}{l}0.0234, \\
{[0.0232,0.0235]}\end{array}$ & $\begin{array}{l}0.0144, \\
{[0.0143,0.0145]}\end{array}$ & $\begin{array}{l}-0.0328, \\
{[-0.0329,-0.0327]}\end{array}$ \\
\hline$\alpha$ & {$[8,13]$} & $\begin{array}{l}-0.0070, \\
{[-0.0070,-0.0068]}\end{array}$ & $\begin{array}{l}0.0351, \\
{[0.0350,0.0353]}\end{array}$ & $\begin{array}{l}-0.0044, \\
{[-0.0044,-0.0043]}\end{array}$ & $\begin{array}{l}0.0037, \\
{[0.0036,0.0038]}\end{array}$ \\
\hline$\beta$ & {$[13,30]$} & $\begin{array}{l}-0.0110, \\
{[-0.0111,-0.0109]}\end{array}$ & $\begin{array}{l}0.0438, \\
{[0.0436,0.0440]}\end{array}$ & $\begin{array}{l}0.0053, \\
{[0.0052,0.0054]}\end{array}$ & $\begin{array}{l}0.0166, \\
{[0.0165,0.0167]}\end{array}$ \\
\hline Lower $\gamma$ & {$[30,45]$} & $\begin{array}{l}0.0031, \\
{[0.0030,0.0032]}\end{array}$ & $\begin{array}{l}0.0263, \\
{[0.0261,0.0265]}\end{array}$ & $\begin{array}{l}0.0287, \\
{[0.0286,0.0288]}\end{array}$ & $\begin{array}{l}0.0145, \\
{[0.0145,0.0146]}\end{array}$ \\
\hline
\end{tabular}


Table 6

Median difference and 95\% CI between pre-seizure and non-seizure EEG.

\begin{tabular}{|c|c|c|c|c|c|}
\hline \multirow{2}{*}{$\begin{array}{l}\text { Frequency } \\
\text { bands }(\mathrm{Hz})\end{array}$} & & \multicolumn{4}{|l|}{ Pre-SEZ vs. Non-SEZ } \\
\hline & & SP & SPWA & WA & EMG \\
\hline$\delta$ & {$[0.5,4]$} & $\begin{array}{l}-0.0107 \\
{[-0.0108,-0.0106]}\end{array}$ & $\begin{array}{l}-0.0241, \\
{[-0.0242,-0.0240]}\end{array}$ & $\begin{array}{l}0.0052, \\
{[0.0051,0.0053]}\end{array}$ & $\begin{array}{l}-0.0123, \\
{[-0.0124,-0.0123]}\end{array}$ \\
\hline$\theta$ & {$[4,8]$} & $\begin{array}{l}-0.0077 \\
{[-0.0077,-0.0076]}\end{array}$ & $\begin{array}{l}-0.0287 \\
{[-0.0288,-0.0287]}\end{array}$ & $\begin{array}{l}0.0050, \\
{[0.0049,0.0050]}\end{array}$ & $\begin{array}{l}-0.0030, \\
{[-0.0030,-0.0030]}\end{array}$ \\
\hline$\alpha$ & {$[8,13]$} & $\begin{array}{l}-0.0115 \\
{[-0.0116,-0.0114]}\end{array}$ & $\begin{array}{l}-0.0365 \\
{[-0.0365,-0.0364]}\end{array}$ & $\begin{array}{l}0.0052, \\
{[0.0051,0.0052]}\end{array}$ & $\begin{array}{l}-0.0017, \\
{[-0.0018,-0.0016]}\end{array}$ \\
\hline$\beta$ & {$[13,30]$} & $\begin{array}{l}-0.0175 \\
{[-0.0175,-0.0174]}\end{array}$ & $\begin{array}{l}-0.0277 \\
{[-0.0278,-0.0276]}\end{array}$ & $\begin{array}{l}-0.0038 \\
{[-0.0039,-0.0037]}\end{array}$ & $\begin{array}{l}-0.0039, \\
{[-0.0040,-0.0038]}\end{array}$ \\
\hline Lower $\gamma$ & {$[30,45]$} & $\begin{array}{l}-0.0129, \\
{[-0.0130,-0.0129]}\end{array}$ & $\begin{array}{l}-0.0097 \\
{[-0.0098,-0.0096]}\end{array}$ & $\begin{array}{l}-0.0069 \\
{[-0.0069,-0.0068]}\end{array}$ & $\begin{array}{l}-0.0039, \\
{[-0.0040,-0.0039]}\end{array}$ \\
\hline
\end{tabular}

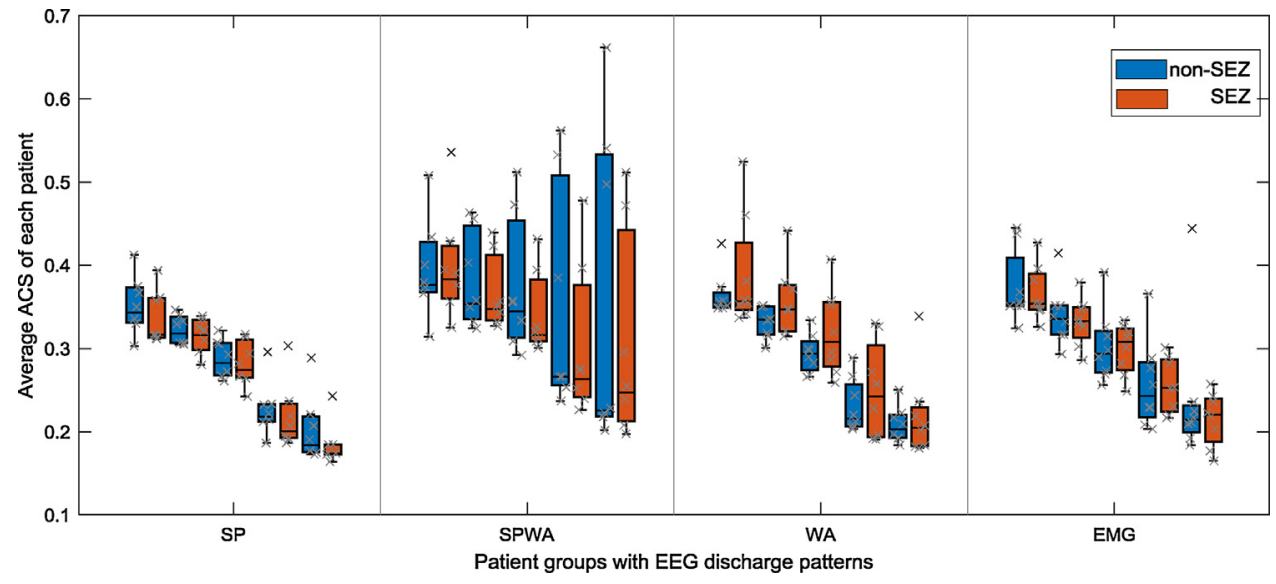

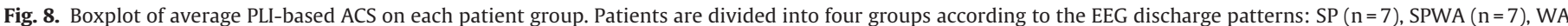

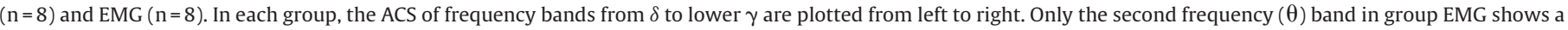
significant difference between non-seizure and seizure EEG $(p=0.0379)$, while other pairs show no significant difference $(p>0.05)$.

because in general, it is more difficult for two high-frequency oscillators without synchronized status to keep a small phase difference in faster trajectories.

\section{Discussion}

CPR can provide additional information (i.e., chaotic PS) compared to PLI by measuring both the linear and nonlinear coherence. The PS measures based on both CPR and PLI methods show significant differences between the awake and sleep EEG backgrounds, and between seizure and non-seizure EEG signals. Both methods can separate non-seizure and pre-seizure EEGs ( 60 s before seizure onsets) for the seizure patterns of SPWA and EMG, which shows the possibility of seizure prediction. The fact that $\mathrm{p}$ values (SEZ vs. Non-SEZ) using CPR is much smaller than that using PLI (see Tables 3 and 4) indicate that CPR may outperform PLI for the seizure detection. However, the PLI method shows a significant difference between non-seizure and pre-seizure EEGs for the SP and WA seizures on specific frequency bands, while the CPR method shows no significant difference. On one hand, it means that PLI may still have an advantage on predicting minor seizures with SP and WA patterns. On the other hand, the performance of CPR method can be further improved by optimizing the parameters chosen. Three important parameters of CPR are the embedding dimension $m$, the time delay $d$, and the coefficient threshold $T$. The two parameters $m$ and $d$ should be estimated in combination (considering $m * d$ product) according to specific EEG signals, e.g., the minimum span of a seizure spike [44]. In this work, the value of threshold $T$ was empirically chosen as $T=0.2$ for all seizure patterns. However, the value of $T$ can be further optimized according to specific seizure discharge patterns. This can be done by a cross validation on a training dataset of each seizure pattern and will be applied in an extended dataset in our future study.

CPR-based ACS decreases in seizures with SP and EMG, while it increases in SPWA seizures, compared with background EEG. The EEG discharge pattern of SP often occurs at the seizure beginning with low-amplitude and increased-frequency signals, referred to as fast intracerebral EEG activity [51] or electrodecremental event [52]. The signals are significantly de-synchronized during the SP seizures [51]. Therefore, the SP seizures show significantly decreased ACS. The EMG seizure patterns occur often in motor seizures such as tonic and myoclonic seizures in the ID patients. It shows significantly decreased PS, which indicates that the functional connectivity of different brain areas is lower during seizures. The EEG discharge pattern of SPWA often occurs in seizures with hyper-synchronous discharges, existing in most absence seizures, e.g., $3-5 \mathrm{~Hz}$ in intellectually normal people, approximately $1.5-3 \mathrm{~Hz}$ in patients with ID. Therefore, ACS based on both CPR and PLI methods show significant increases during seizures. The discharge pattern of WA often corresponds to short and focal EEG discharges, which are associated with clinically minor seizures that are difficult to detect [22]. Since focal seizures often affect a smaller brain region, the changes of ACS (i.e., average PS level) may be not significant across patients. Another reason may be the regular occurrence of a slow activity (i.e., seizure imitators) in the background EEG of patients with an ID [26].

This study proposed a new method to generate a brain FCN and showed that the ACS of the brain FCN is able to discriminate seizure 
EEG signals. In addition, structural network differences may exist among patients with different ID levels [53]. The brain FCN might disclose more underlying epileptic information such as the functional modularity of brains [6] by using network theory [1] than only using traditional analysis methods. The brain FCN with fully connected edges can be mapped into a binary FCN with partial connections. The method to infer whether or not it is a synchronized status (which determines a functional connection edge 0 or 1 ) in an ambiguous range of PS was further developed [36]. In a binary FCN, network topologies such as the average cluster coefficient (ACC) [54] and assortativity coefficient (AC) [55] can be computed. More network topologies derived from EEG signals can be found in [27] [28]. These network topologies can be further used to detect a generalized seizure [27] or locate the onset zone for a focal seizure [56].

\section{Conclusion}

The CPR method measures PS of a pair of EEG signals and provides a new method for modeling the epilepsy-related brain functional network. Compared with the traditional PLI method, CPR can directly measure broadband EEG signals and captures nonlinear coupling. Both CPR and PLI methods show that the background EEG in awake and sleep status have different baselines of PS in all ID patients. Smaller p values indicate that CPR may outperform PLI in the seizure detection (i.e., discriminating seizure EEG from nonseizure backgrounds). PS changes in seizures depend on the EEG discharge patterns. CPR-based PS decreases significantly in seizures with fast spike and seizures with EMG activity, while increases in the spike-wave seizures. In addition, CPR-based PS shows potential possibility in predicting the spike-wave seizures and seizure with EMG activities in an early manner.

\section{Appendix A.}

See Figs. A1-A3.
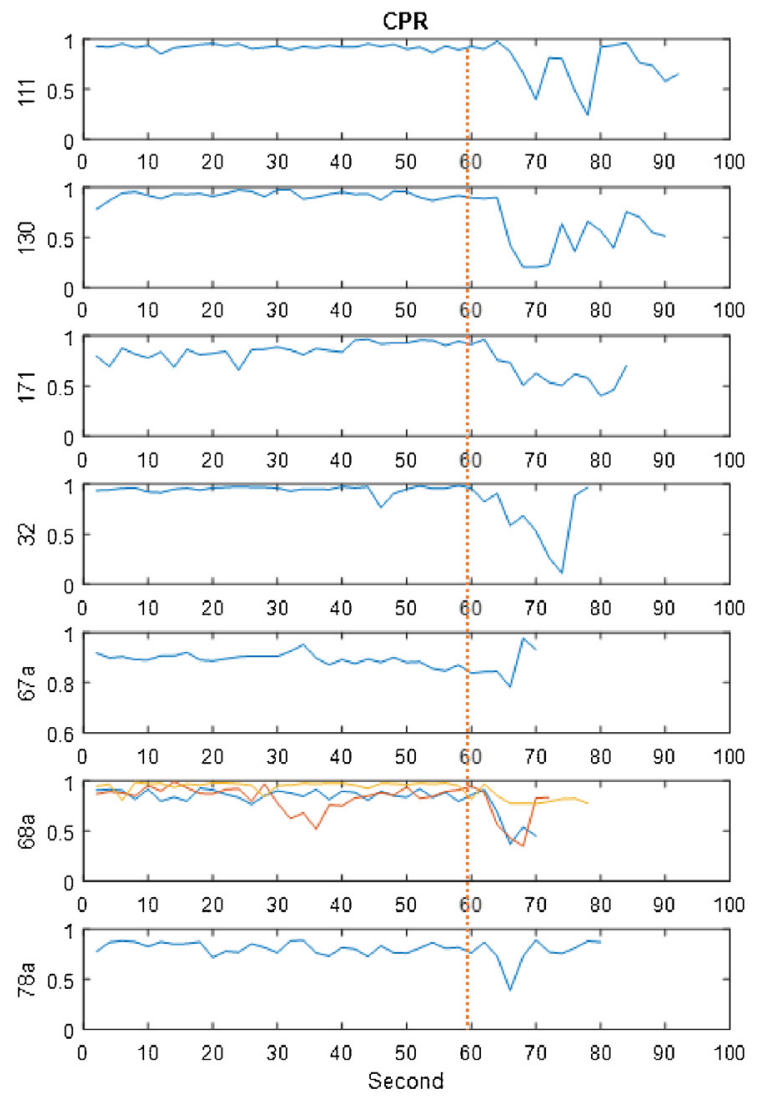
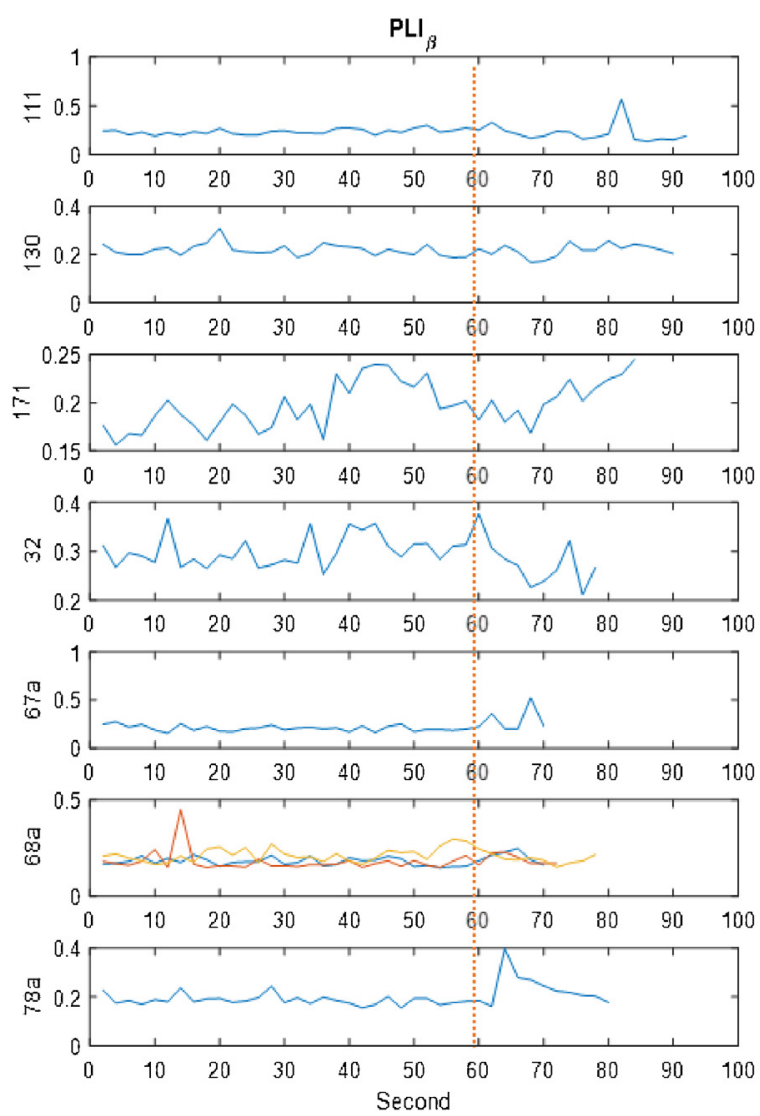

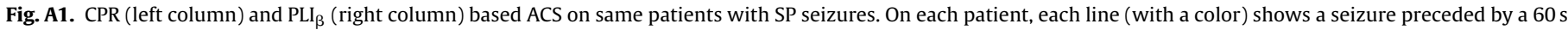
non-seizure EEG till seizure offsets. The dash lines show the aligned seizures onsets. 

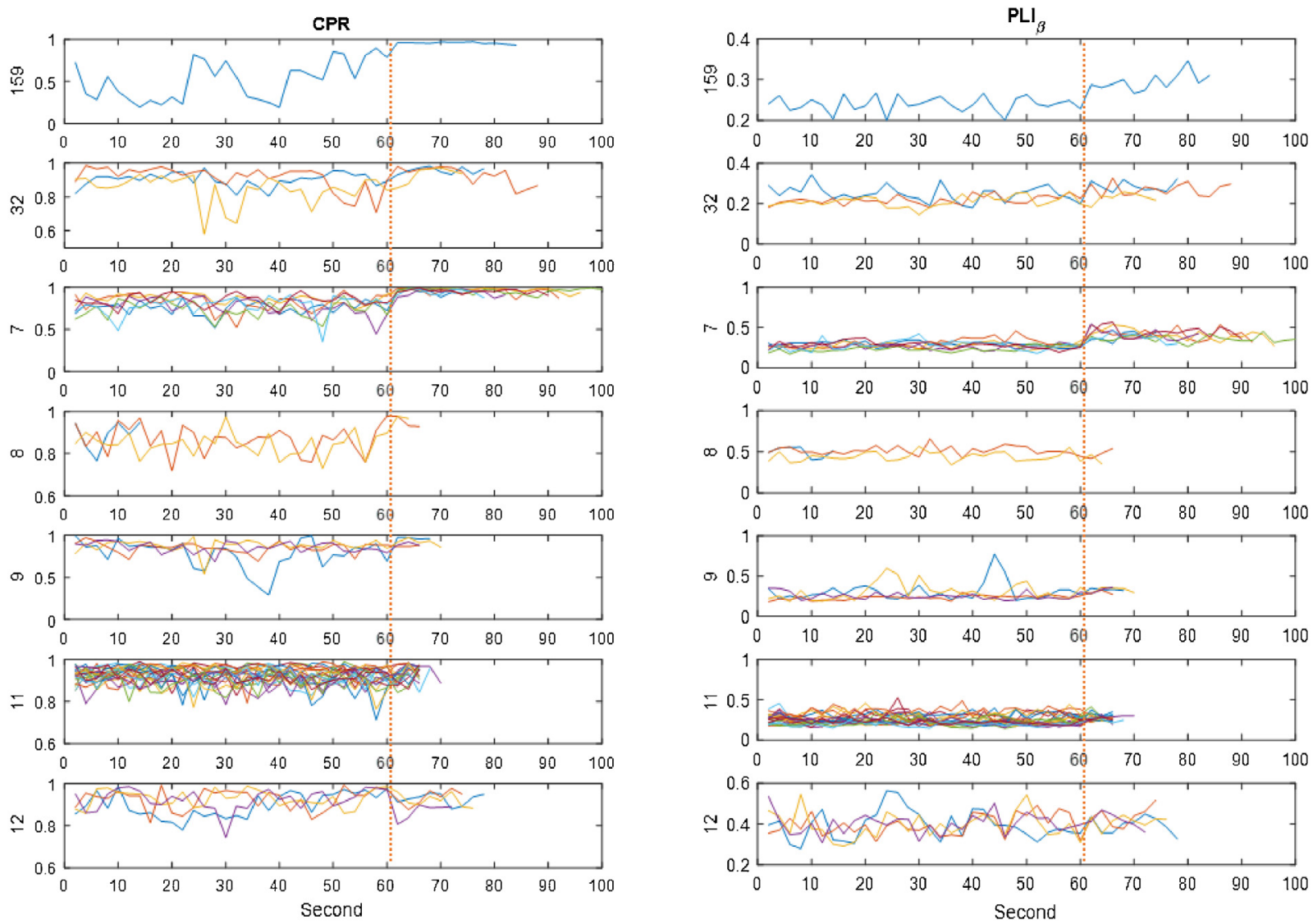

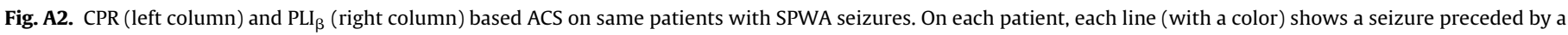
60 s non-seizure EEG till seizure offsets. The dash lines show the aligned seizures onsets. 

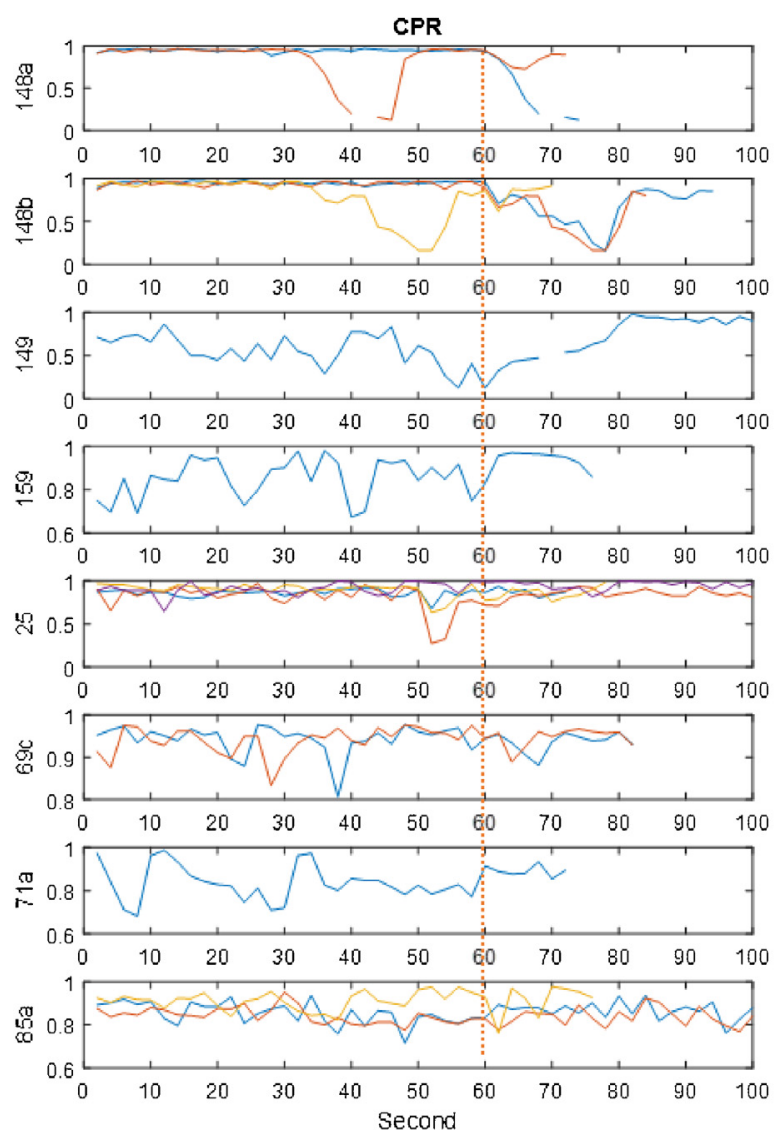
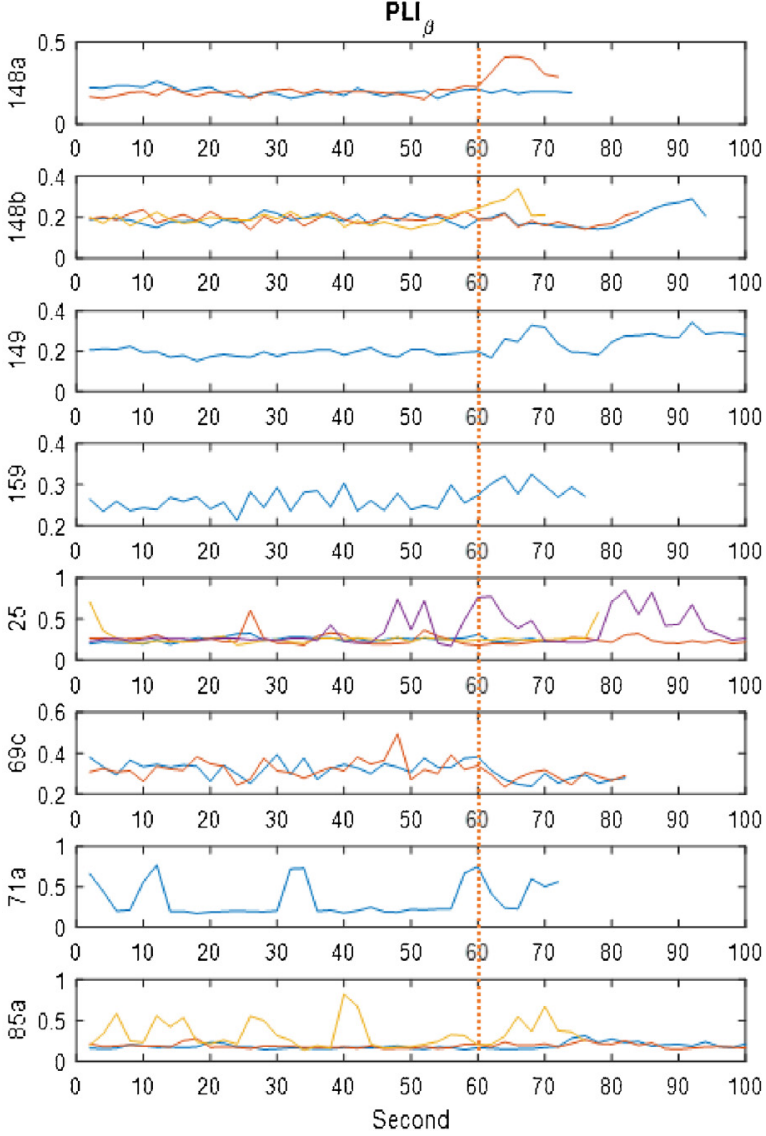

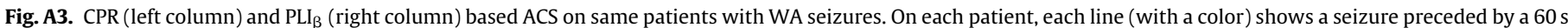
non-seizure EEG till seizure offsets. The dash lines show the aligned seizures onsets.

\section{Appendix B.}

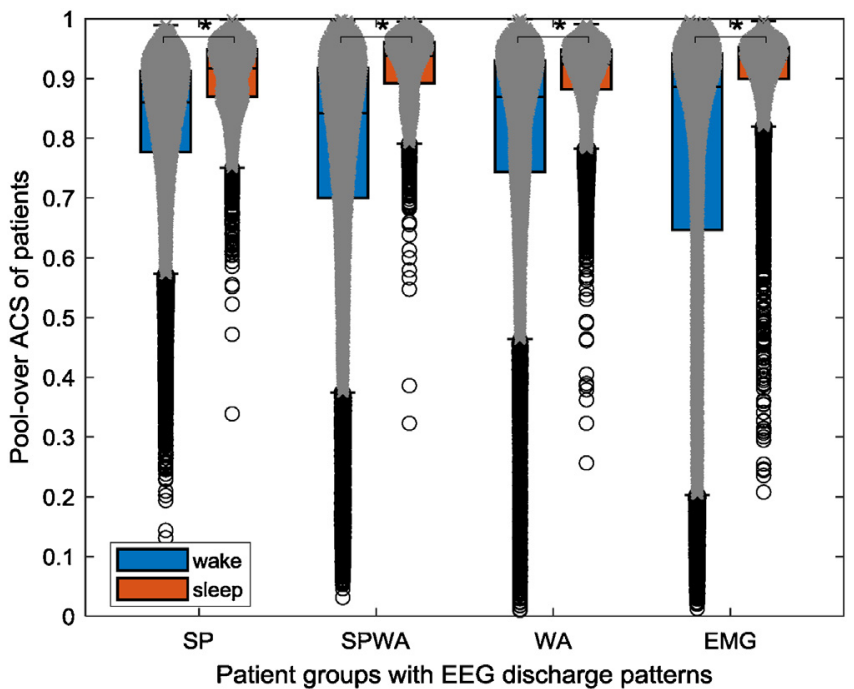

Fig. B1. Boxplot of CPR-based ACS on each patient group. Patients are divided into four groups according to the EEG discharge patterns: $S P\left(n_{0}=12600, n_{1}=85\right)$, SPWA $\left(\mathrm{n}_{0}=12600, \mathrm{n}_{1}=250\right)$, WA $\left(\mathrm{n}_{0}=14400, \mathrm{n}_{1}=525\right)$, and EMG $\left(\mathrm{n}_{0}=14400, \mathrm{n}_{1}=568\right)$, where $n_{0}$ is the size of non-seizure class (non-SEZ), $n_{1}$ is the size of seizure class (SEZ). The * denotes significant difference between awake and sleep EEG ( $p<1.000 \mathrm{e}-255)$.

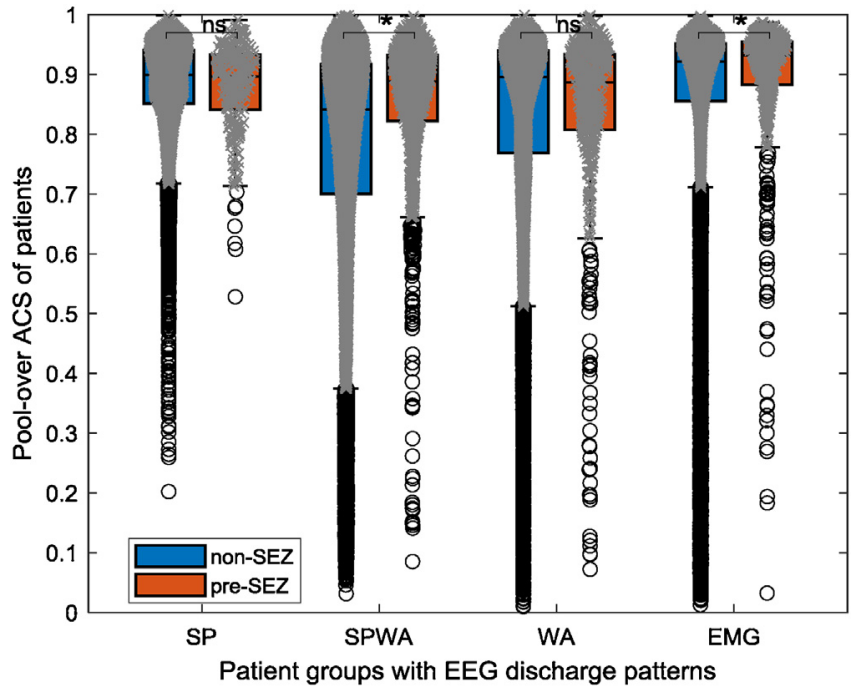

Fig. B2. Boxplot of CPR-based ACS on each patient group. Patients are divided into four groups according to the EEG discharge patterns SP $\left(\mathrm{n}_{0}=12600, \mathrm{n}_{1}=85\right)$, SPWA $\left(\mathrm{n}_{0}=12600, \mathrm{n}_{1}=250\right)$, WA $\left(\mathrm{n}_{0}=14400, \mathrm{n}_{1}=525\right)$, and EMG $\left(\mathrm{n}_{0}=14400, \mathrm{n}_{1}=568\right)$, where $n_{0}$ is the size of non-seizure class (non-SEZ), $n_{1}$ is the size of seizure class (SEZ). The * denotes significant difference; ns denotes not significant (specific $\mathrm{p}$ values refer to Table 3 ). 


\section{References}

[1] F. Varela, J.-P. Lachaux, E. Rodriguez, J. Martinerie, The brainweb: phase synchronization and large-scale integration, Nat. Rev. Neurosci. 2 (2001) 229

[2] L. Douw, M. de Groot, E. van Dellen, J.J. Heimans, H.E. Ronner, C.J. Stam, J.C. Reijneveld, Functional connectivity is a sensitive predictor of epilepsy diagnosis after the first seizure, PLoS One 5 (2010) 1-7.

[3] K. Lehnertz, S. Bialonski, M.-T. Horstmann, D. Krug, A. Rothkegel, Mu. Staniek T. Wagner, Synchronization phenomena in human epileptic brain networks, J. Neurosci. Methods 183 (2009) 42-48.

[4] A. Sazonov, C. Ho, J.M. Bergmans, J.A.M. Arends, P.M. Griep, E. Verbitskiy, P.M Cluitmans, P.J.M. Boon, An investigation of the phase locking index for measuring of interdependency of cortical source signals recorded in the EEG, Biol. Cybern. 100 (2009) 129-146.

[5] F. Mormann, T. Kreuz, C. Rieke, R.G. Andrzejak, A. Kraskov, P. David, C.E. Elger, K. Lehnertz, On the predictability of epileptic seizures, Clin. Neurophysiol. 116 (2005) 569-587.

[6] M. Chavez, M. Valencia, V. Navarro, V. Latora, J. Martinerie, Functional modularity of background activities in normal and epileptic brain networks, Phys. Rev. Lett. 104 (2010), 118701.

[7] M.A. Quraan, C. McCormick, M. Cohn, T.A. Valiante, M.P. McAndrews, Altered resting state brain dynamics in temporal lobe epilepsy can Be observed in spectral power, functional connectivity and graph theory metrics, PLoS One 8 (2013), e68609.

[8] E. Pereda, R.Q. Quiroga, J. Bhattacharya, Nonlinear multivariate analysis of neurophysiological signals, Prog. Neurobiol. 77 (2005) 1-37.

[9] K. Ansari-Asl, L. Senhadji, J.-J. Bellanger, F. Wendling, Quantitative evaluation of linear and nonlinear methods characterizing interdependencies between brain signals, Phys. Rev. E 74 (2006), 031916.

[10] R.G.A.F. Mormann, C.E. Elger, K. Lenhnertz, Seizure prediction: the long and the winding road, Brain 130 (2) (2007) 314-333.

[11] K.J. Friston, Book review: brain function, nonlinear coupling, and neuronal transients, Neuroscientist 7 (2001) 406-418.

[12] H. Korn, P. Faure, Is there chaos in the brain? II. Experimental evidence and related models, C. R. Biol. 326 (2003) 787-840.

[13] R. Acharya, O. Faust, N. Kannathal, T. Chua, S. Laxminarayan, Non-linear analysis of EEG signals at various sleep stages, Comput. Methods Programs Biomed. 80 (2005) 37-45.

[14] Y. Yuan, Y. Li, D.P. Mandic, A comparison analysis of embedding dimensions between normal and epileptic EEG time series, J. Physiol. Sci. 58 (2008) 239-247.

[15] S. Sarbadhikari, K. Chakrabarty, Chaos in the brain: a short review alluding to epilepsy, depression, exercise and lateralization, Med. Eng. Phys. 23 (2001) 447-457.

[16] F. Mormann, K. Lehnertz, P. David, C.E. Elger, Mean phase coherence as a measure for phase synchronization and its application to the EEG of epilepsy patients, Physica D: Nonlinear Phenomena 144 (2000) 358-369.

[17] A. Schad, K. Schindler, Br. Schelter, T. Maiwald, A. Brandt, J. Timmer, A. Schulze-Bonhage, Application of a multivariate seizure detection and prediction method to non-invasive and intracranial long-term EEG recordings, Clin. Neurophysiol. 119 (2008) 197-211.

[18] M. Chavez, M. Le Van Quyen, V. Navarro, M. Baulac, J. Martinerie, Spatio-temporal dynamics prior to neocortical seizures: amplitude versus phase couplings, IEEE Trans. Biomed. Eng. 50 (2003) 571-583.

[19] M.L.V. Quyen, J. Foucher, J.-P. Lachaux, E. Rodriguez, A. Lutz, J. Martinerie, FJ. Varela, Comparison of Hilbert transform and wavelet methods for the analysis of neuronal synchrony, J. Neurosci. Methods 111 (2001) 83-98.

[20] U.R. Acharya, S.V. Sree, G. Swapna, R.J. Martis, J.S. Suri, Automated EEG analysis of epilepsy: a review, Knowledge Based Syst. 45 (2013) 147-165.

[21] E.H. Bertram, Temporal lobe epilepsy: where do the seizures really begin? Epilepsy Behav. 14 (2009) 32-37.

[22] L. Wang, J.B.A.M. Arends, X. Long, P.J.M. Cluitmans, J.P. van Dijk, Seizure pattern-specific epileptic epoch detection in patients with intellectual disability, Biomed. Signal Process. Control 35 (2017) 38-49.

[23] T. Matthews, N. Weston, H. Baxter, D. Felce, M. Kerr, A general practice-based prevalence study of epilepsy among adults with intellectual disabilities and of its association with psychiatric disorder, behaviour disturbance and carer stress, J. Intellect. Disabil. Res. 52 (2008) 163-173.

[24] J.S. van Ool, F.M. Snoeijen-Schouwenaars, H.J. Schelhaas, I.Y. Tan, A.P. Aldenkamp, J.G.M. Hendriksen, A systematic review of neuropsychiatric comorbidities in patients with both epilepsy and intellectual disability, Epilepsy Behav. 60 (2016) 130-137.

[25] U. Steffenburg, A. Hedström, A. Lindroth, L.M. Wiklund, G. Hagberg, M. Kyllerman, Intractable epilepsy in a population-based series of mentally retarded children, Epilepsia 39 (1998) 767-775.

[26] L. Wang, X. Long, J.B.A.M. Arends, J.P. van Dijk, R.M. Aarts, EEG-based Seizure Detection in Patients with Intellectual Disability: which EEG and clinical factors are important? Biomed. Signal Process. Control (2018), In submission.

[27] L. Wang, X. Long, J.B.A.M. Arends, R.M. Aarts, EEG analysis of seizure patterns using visibility graphs for detection of generalized seizures, J. Neurosci. Methods 290 (2017) 85-94
[28] S. Sargolzaei, M. Cabrerizo, M. Goryawala, A.S. Eddin, M. Adjouadi, Scalp EEC brain functional connectivity networks in pediatric epilepsy, Comput. Biol. Med. 56 (2015) 158-166.

[29] K. Abualsaud, M. Mahmuddin, M. Saleh, A. Mohamed, Ensemble classifier for epileptic seizure detection for imperfect EEG data, The Scientific World Journal 2015 (2015).

[30] P. Thodoroff, J. Pineau, A. Lim, Learning robust features using deep learning for automatic seizure detection, in: Machine Learning for Healthcare Conference, 2016, pp. 178-190.

[31] D. Rangaprakash, N. Pradhan, Study of phase synchronization in multichannel seizure EEG using nonlinear recurrence measure, Biomed. Signal Process. Control 11 (2014) 114-122.

[32] C. Bandt, A. Groth, N. Marwan, M.C. Romano, M. Thiel, M. Rosenblum, Ju. Kurths, Mathematical Methods in Signal Processing and Digital Image Analysis, rgen, Analysis of bivariate coupling by means of recurrence, Springer, 2008, pp. 153-182.

[33] N. Marwan, M. Carmen Romano, M. Thiel, J. Kurths, Recurrence plots for the analysis of complex systems, Phys. Rep. 438 (2007) 237-329.

[34] C.J. Stam, Nonlinear dynamical analysis of EEG and MEG: review of an emerging field, Clin. Neurophysiol. 116 (2005) 2266-2301.

[35] J. Schumacher, R. Haslinger, G. Pipa, A statistical modeling approach for detecting generalized synchronization, Phys. Rev. E Stat. Nonlinear Soft Matter Physics 85 (2012), 056215-056215.

[36] D. Rangaprakash, Binarized brain connectivity: a novel autocorrelation-based iterative synchronization technique, IEEE Trans. Signal Inf. Process. Netw 3 (2017) 660-668

[37] D. Rangaprakash, X. Hu, G. Deshpande, Phase synchronization in brain networks derived from correlation between probabilities of recurrences in functional MRI data, Int. J. Neural Syst. 23 (2013), 1350003.

[38] M.G. Kitzbichler, M.L. Smith, So. Christensen, R. ren, E. Bullmore, Broadband criticality of human brain network synchronization, PLoS Comput. Biol. 5 (2009), e1000314.

[39] R. Blasco, M. Carmen, Synchronization Analysis by Means of Recurrences in Phase Space, 1, Universitat sbibliothek, 2004, PhD thesis.

[40] B.M. Altevogt, H.R. Colten, Sleep Disorders and Sleep Deprivation: an Unmet Public Health Problem, National Academies Press, 2006.

[41] S. Schiff, Dangerous phase, Neuroinformatics 3 (2005) 315-317.

[42] F. Takens, Detecting Strange Attractors in Turbulence, in: Dynamical Systems and Turbulence, 1981, Springer, Warwick, 1980, pp. 366-381.

[43] D.P. Subha, P.K. Joseph, R. Acharya, C.M. Lim, EEG signal analysis: a survey, J. Med. Syst. 34 (2010) 195-212.

[44] L.M. Pecora, L. Moniz, J. Nichols, T.L. Carroll, A unified approach to attractor reconstruction, Chaos Interdiscip. J. Nonlinear Sci. 17 (2007), 013110.

[45] J. Kurths, M.C. Romano, M. Thiel, G.V. Osipov, M.V. Ivanchenko, I.Z. Kiss, J.L. Hudson, Synchronization Analysis of Coupled Noncoherent Oscillators, Nonlinear Dyn. 44 (2006) 135-149.

[46] N.E. Huang, Z. Shen, S.R. Long, M.C. Wu, H.H. Shih, Q. Zheng, N.-C. Yen, C.C. Tung, H.H. Liu, The empirical mode decomposition and the Hilbert spectrum for nonlinear and non-stationary time series analysis, in: Proceedings of the Royal Society of London A: Mathematical, Physical and Engineering Sciences, 1998, pp. 903-995.

[47] J.-P. Lachaux, E. Rodriguez, J. Martinerie, F.J. Varela, Measuring phase synchrony in brain signals, Hum. Brain Mapp. 8 (1999) 194-208.

[48] T.M.E. Nijsen, J.B.A.M. Arends, P.A.M. Griep, P.J.M. Cluitmans, The potential value of three-dimensional accelerometry for detection of motor seizures in severe epilepsy, Epilepsy Behav. 7 (2005) 74-84.

[49] S.J. Mason, N.E. Graham, Areas beneath the relative operating characteristics (ROC) and relative operating levels (ROL) curves: statistical significance and interpretation, Q. J. R. Meteorol. Soc. 128 (2002) 2145-2166.

[50] L. Dümbgen, B.-W. Igl, A. Munk, P-values for classification, Electron. J. Stat. 2 (2008) 468-493.

[51] F. Wendling, F. Bartolomei, J.-J. Bellanger, J. Bourien, P. Chauvel, Epileptic fast intracerebral EEG activity: evidence for spatial decorrelation at seizure onset, Brain 126 (2003) 1449-1459.

[52] K.K. Jerger, T.I. Netoff, J.T. Francis, T. Sauer, L. Pecora, S.L. Weinstein, S.J. Schiff, Early seizure detection, J. Clin. Neurophysiol. 18 (2001) 259-268.

[53] S. Gu, F. Pasqualetti, M. Cieslak, Q.K. Telesford, A.B. Yu, A.E. Kahn, J.D. Medaglia, J.M. Vettel, M.B. Miller, S.T. Grafton, D.S. Bassett, Controllability of structural brain networks, Nat. Commun. 6 (2015) 8414.

[54] Eb. Ravasz, A.L. Somera, D.A. Mongru, Z.N. Oltvai, A. Barab, Hierarchical organization of modularity in metabolic networks, Science 297 (2002) 1551-1555.

[55] M.E.J. Newman, Assortative mixing in networks, Phys. Rev. Lett. 89 (2002), 208701.

[56] S.P. Burns, S. Santaniello, R.B. Yaffe, C.C. Jouny, N.E. Crone, G.K. Bergey, W.S. Anderson, S.V. Sarma, Network dynamics of the brain and influence of the epileptic seizure onset zone, Proceedings of the National Academy of Sciences 111 (2014) E5321-E5330. 